\title{
Unternehmertum jenseits des Normalunternehmertums: Für eine praxistheoretisch inspirierte Erforschung unternehmerischer Aktivitäten
}

\author{
Andrea D. Bührmann
}

Zusammenfassung: Am Beispiel der gegenwärtig zu beobachtenden Prekarisierung des Arbeitens wird dargelegt, worin der „Mehrwert“ eines um arbeitssoziologische Erkenntnisse erweiterten Blicks auf unternehmerische Aktivitäten besteht. Zunächst wird der Forschungsstand zu den Prekarisierungsprozessen von abhängiger Erwerbsarbeit rekonstruiert. Deutlich wird, dass zwar intensiv über die Erosion des sogenannten Normalarbeitsverhältnisses und dann über eine fortschreitende Prekarisierung von abhängiger Erwerbstätigkeit diskutiert worden ist. Jedoch ist die Frage nach einer Prekarisierung des unternehmerischen Arbeitens bislang weitgehend ausgeblendet geblieben. Dass und inwiefern auch in Bezug auf dieses Arbeiten eine zunehmende Prekarisierung zu beobachten ist, wird in einem zweiten Schritt erörtert. Zudem werden zentrale Ursachenkomplexe für Prekarisierungsprozesse unternehmerischer Aktivitäten skizziert. Im Anschluss wird zum einen nach den Implikationen einer ,doppelten“ Prekarisierung von Arbeit und deren gesellschaftlichen Vermittlungen gefragt. Zum anderen wird das methodisch-methodologische Potenzial einer praxistheoretischen Fundierung der Forschung aufgezeigt.

Schlüsselwörter: Erosion der Normalarbeit · Unternehmer und Unternehmerinnen · Prekarität · Praxisforschung

\section{Towards a casualization of corporate activities? A plea for practice inspired research on the casualization of labor}

\begin{abstract}
Using the example of the present casualization of labor the following article demonstrates the "surplus value" of a perspective on corporate activities that is enriched by insights gained from the field of the sociology of labor. Beginning with a reconstruction of the state of the art on casualization processes of dependent labor it is shown that there has indeed been an intense discussion about the erosion of the so-called standard employment relationship (Normalarbeitsverhältnis) and the advancing casualization of dependent labor. The question regarding the casualization of the corporate activities, however, has hardly been recognized. The article will
\end{abstract}

(C) Die Autor(en) 2012. Dieser Artikel ist auf Springerlink.com mit Open Access verfügbar.

\footnotetext{
A. D. Bührmann $(\bowtie)$

Institut für Soziologie, Universität Göttingen,

Platz der Göttinger Sieben 3, 37073 Göttingen, Deutschland

E-Mail: andrea.buehrmann@uni-goettingen.de
} 
demonstrate that there actually is an ongoing casualization of corporate activities. Moreover, the vital factors for casualization processes in corporate activities will be elaborated. Subsequent to these points, the text raises the question of the implications of a "twofold" casualization of labor and its social mediations. In a final step, the article focuses on the systematic-methodological potential of founding research in a theory of practice.

Keywords: Erosion of the standard employment contract - Casualization · Entrepreneurs · Praxis research

\section{L'entrepreneuriat au-delà de l'entrepreneuriat normal: Pour une étude des activités entrepreneuriales inspirée de la théorie de la pratique}

Résumé: Cet article montre à partir de l'exemple de la précarisation du travail observable actuellement ce que l'étude des activités entrepreneuriales peut apprendre de la sociologie du travail. Il est en premier lieu fait état des recherches sur les processus de précarisation du travail salarié. Il apparaît ainsi que l'érosion de ce que l'on appelle l'emploi salarié normal et la précarisation croissante du travail salarié font l'objet d'intenses discussions. L'hypothèse d'une précarisation du travail entrepreneurial est cependant restée largement ignorée. Dans un deuxième temps, cet article discute si et dans quelle mesure une précarisation croissante peut être également observée dans le cadre de cette forme de travail. Il est en outre fait mention des principales causes des processus de précarisation des activités entrepreneuriales. Enfin, cet article s'interroge sur les implications d'une «double» précarisation du travail et sur ses mécanismes sociaux avant de montrer les potentialités méthodiques et méthodologiques de la théorie de la pratique pour la recherche sur les activités entrepreneuriales.

Mots-clés: Érosion du travail normal · Entrepreneurs et entrepreneuses · Précarité ·

Recherche sur la pratique

\section{Einleitung}

Nicht zuletzt angesichts der aktuellen Weltwirtschaftskrise erlebt die Wirtschaftssoziologie derzeit eine Renaissance. Ihre Befunde, Konzepte und theoretischen Einsichten werden zunehmend auch außerhalb der einschlägigen Expertenzirkel rezipiert (vgl. dazu ausführlich Maurer 2008; Smelser und Swedberg 2005; Swedberg und Granovetter 2001). Inmitten dieser Renaissance bleibt indes - zumindest bisher - die Forschung über die unterschiedlichen Formen unternehmerischer Aktivitäten merkwürdig blass, obgleich ihre Zahl in den letzten Jahren erheblich zugenommen hat - so sind 2010 über 4,4 Mio. Personen unternehmerisch tätig gewesen - und sich das Feld unternehmerischer Aktivitäten zunehmend diversifiziert hat; das Spektrum reicht z.B. vom alteingesessenen Mittelständler aus der Bourgeoisie über die erfolgreiche Unternehmensberaterin bis hin zum „kleinen“ Selbstständigen. Zwar wurde der Begriff der unternehmerischen Selbstständigkeit ausgeweitet. Ein instruktives Beispiel ist die Definition des Mikrozensus, in dem Selbstständige als Personen definiert werden, „die eine auf wirtschaftlichen Erwerb gerichtete Tätigkeit ausüben, unabhängig vom Umfang dieser Tätigkeit“" (Statistisches 
Bundesamt 2011). ${ }^{1}$ Damit wird nicht nur explizit der Umfang der unternehmerischen Aktivitäten nicht näher bestimmt, sondern auch implizit nicht vorausgesetzt, dass Unternehmer wie Unternehmerinnen auch andere als sich selbst in ihrem Betrieb beschäftigen. Diese definitorische Ausweitung des Begriffs vom Unternehmertum ist jedoch paradoxerweise von einer forschungspraktischen Verkürzung begleitet. So fokussiert man auch in der soziologischen Unternehmensforschung bisher - vielfach ausgehend von modelltheoretischen Überlegungen aus der Ökonomie - auf die Erforschung erfolgreichen, d. h. eines kapitalintensiven und wachstumsorientierten Unternehmertums, das Arbeitsplätze schafft und erhält sowie vor allen Dingen auch den Unternehmern und Unternehmerinnen selbst ein gesichertes Einkommen verspricht (vgl. dazu Aldrich 2005; Maurer und Schimank 2008; Swedberg 2009): Während sich die einen im Rekurs auf (neo-)marxistische Positionen in einer vor allen Dingen makrosoziologischen Perspektive damit begnügen, Unternehmer als „Personifizierung des Kapitals“ oder auch als bloße „Agenten“ kapitalistischer Bewegungsgesetze zu thematisieren, konzentrieren sich andere im Anschluss an Joseph A. Schumpeters $(1928,1993)$ Konzept des Unternehmers als ,,innovativen Zerstörer" auf die Erfolgsbedingungen unternehmerischen Entscheidens und Handelns (vgl. für einen Überblick über die Diskussion Becker und Knudsen 2003). Obwohl nun etwa Christoph Deutschmann (2008) solche mikrosoziologischen Konzeptionen als unterkomplex kritisiert hat - sie reduzierten Selbstständige im Grunde auf ,informationsverarbeitende Maschinen“, die stets kühl kalkulierend den Gesetzen der Profitmaximierung folgten -, verkörpert doch auch für ihn das Unternehmertum ,einen neuen, auf Geld und Markterfolg gegründeten Typus sozialer Aufwärtsmobilität, der zum Motor nicht nur wirtschaftlicher, sondern gesamtgesellschaftlicher Transformationen wird und einen anhaltenden institutionellen Veränderungsdruck bewirkt“" (ebd., S. 116 f.). Und Werner Rammert (2008) betont z. B. das charismatische Potenzial des Unternehmerischen. In beiden Debattenbeiträgen - die hier beispielhaft für viele andere stehen - geht es um die Erfolgspotenziale unternehmerischer Aktivitäten. Sie interessieren sich kaum für die Bedingungen des bloßen unternehmerischen Überlebens. Pointiert ausgedrückt beherrscht somit bislang die folgende Forschungsperspektive die soziologisch orientierte Unternehmensforschung: ${ }^{2}$ Unternehmer und Unternehmerinnen sind entweder erfolgreich oder sie scheitern; haben sie indes Erfolg, erreichen sie (mindestens) den gesicherten Mittelstand, scheitern sie, so zählen sie nicht mehr zum Unternehmertum (vgl. dazu auch Bührmann und Pongratz 2010).

Damit folgt die soziologische Unternehmensforschung einem in der volks- bzw. betriebswirtschaftlichen Forschung hervorgebrachten und gebräuchlichen Begriff des Unternehmertums, ohne allerdings dessen Theorieeffekte (Bourdieu 1987, 1990) genügend zu reflektieren: Konzentriert sich die Forschung nämlich auf erfolgreiches Unternehmertum, so abstrahiert sie auch von den anderen, weniger erfolgreichen Praxen unternehmerischer Akteure und (re-)produziert so die Vorstellung eines Normaltunternehmertums. Als „Normalunternehmer“ (Bührmann 2007) gilt demnach, wer

1 Im Folgenden wird zunächst auf diese Definition der Unternehmerin bzw. des Unternehmers abgestellt. Dabei werden die Begriffe „Selbstständige“ und „Unternehmer“ bzw. „Unternehmerin" synonym verwendet.

2 Eine Ausnahme bilden hier die Studien von Bögenhold (1987), Bologna (2006) und Candeias (2008, 2009). 
- erstens ein berufserfahrener, erwerbstätiger Mann ohne Migrationshintergrund ist, der über angemessenes ökonomisches Kapital verfügt und profunde unternehmerische Fachkenntnisse sowie eine entsprechende (Berufs-)Ausbildung hat,

- zweitens sich rast- und ruhelos in Vollzeit seinem Unternehmen widmet und so ein Unternehmenswachstum generiert sowie Arbeitsplätze schafft und schließlich

- drittens aus den erfolgreichen unternehmerischen Aktivitäten ein gesichertes Einkommen erzielen kann.

Dieser Theorie-Effekt kann - soweit ich sehe - auch darauf zurückgeführt werden, dass die Wirtschaftssoziologie insbesondere mit der Arbeitssoziologie bisher noch nicht in einen intensiveren Dialog eingetreten ist und die Forschungsbefunde wechselseitig noch kaum systematisch rezipiert worden sind. Ein solcher Dialog soll mit diesem Beitrag angestoßen werden. Das übergeordnete Ziel besteht darin, die Debatten von Arbeits- und Wirtschaftssoziologie stärker miteinander zu verknüpfen und so füreinander produktiv zu machen.

Den Ausgangspunkt des Beitrages bilden der angesprochene Theorie-Effekt und seine Folgen. Am Beispiel der gegenwärtig zunehmend zu beobachtenden Prekarisierung des Arbeitens soll dargelegt werden, worin der „Mehrwert“ eines um arbeitssoziologische Erkenntnisse erweiterten Blicks auf unternehmerische Aktivitäten besteht. In einem ersten Schritt wird zunächst der aktuelle Forschungsstand zu den Prekarisierungsprozessen von abhängiger Erwerbsarbeit in seinen wesentlichen Grundzügen rekonstruiert. Deutlich wird, dass seit den 1990er Jahren zwar intensiv zunächst über die Erosion des sogenannten Normalarbeitsverhältnisses und dann über eine fortschreitende Prekarisierung von abhängiger Erwerbstätigkeit diskutiert worden ist. Jedoch ist die Frage nach einer möglichen Prekarisierung unternehmerischen Arbeitens bislang weitgehend ausgeblendet geblieben. Dass und inwiefern aber auch in Bezug auf dieses Arbeiten eine zunehmende Prekarisierung zu beobachten ist, wird in einem zweiten Schritt anhand von neueren Untersuchungen aus der Unternehmensforschung erörtert. Hier wird der aktuelle Forschungsstand zur objektiven Prekarisierung und der subjektiv gefühlten Prekarisierung unternehmerischen Arbeitens komprimiert vorgestellt. Zudem werden zentrale Ursachenkomplexe für Prekarisierungsprozesse unternehmerischer Aktivitäten skizziert. Im Anschluss werden dann die theoretischen Konsequenzen und methodischen Herausforderungen dieser Prekarisierungsprozesse diskutiert. Es wird zum einen nach den Implikationen einer - wenn man so will - doppelten Prekarisierung von Arbeit und deren gesellschaftlichen Vermittlungen gefragt. Zum anderen wird das methodisch-methodologische Potenzial einer praxistheoretischen Fundierung der Forschung aufgezeigt. Abschließend werden ein kurzes Fazit gezogen und weitere Forschungsperspektiven skizziert.

\section{Die Erosion des Normalarbeitsverhältnisses und die Prekarisierung abhängiger Erwerbsarbeit}

In der Bundesrepublik Deutschland ist lange Zeit das sogenannte Normalarbeitsverhältnis (Mückenberger 1985) vor allem für Männer ohne Migrationshintergrund die prägende 
Form von Beschäftigungsverhältnissen gewesen. ${ }^{3}$ Es hat sich als typisches Arbeitsverhältnis seit dem 19. Jahrhundert nicht zuletzt aufgrund des Engagements der Gewerkschaften und der Arbeiterbewegung in den westlichen Industriegesellschaften herausgebildet. Spätestens nach dem Einsetzen der Wirtschaftskrise 1973/1974 sind jedoch erste Anzeichen einer Erosion des Normalarbeitsverhältnisses konstatiert worden ${ }^{4}$ : Während seitdem immer weniger Menschen auf unbefristeten und sozialversicherungspflichtigen Vollzeitstellen beschäftigt gewesen sind, ist eine Pluralisierung der Erwerbsformen zu beobachten, und die Zahl derjenigen, die in sogenannten atypischen Beschäftigungsverhältnissen arbeiten, ist stetig angestiegen. ${ }^{5}$

Aktuelle Auswertungen des Mikrozensus verweisen auf eine Erosion des Normalarbeitsverhältnisses: Sie zeigen, ${ }^{6}$ dass 2008 von den „Kernerwerbstätigen ${ }^{67}$ noch $66,0 \%$ (22,9 Mio.) in Normalarbeitsverhältnissen und 22,2\% (7,7 Mio.) in atypischen Beschäftigungsverhältnissen tätig gewesen sind. ${ }^{8}$ Damit hat sich die Struktur der Erwerbstätigen, die abhängig beschäftigt sind, grundlegend verändert. Denn der Anteil der Kernerwerbstätigen, die in Normalarbeitsverhältnissen gearbeitet haben, ist von 1998 bis 2008 um $6,6 \%$ zurückgegangen. Im gleichen Zeitraum erhöhte sich der Anteil der atypischen Beschäftigungsverhältnisse um 6\% (Abb. 1).

Von den erwähnten 22,2\% Kernerwerbstätigen in atypischen Beschäftigungen arbeiteten 2008 7,9\% in befristeten Arbeitsverhältnissen, 14,1\% in Teilzeitbeschäftigungen, ${ }^{9}$ $7,6 \%$ in geringfügigen Beschäftigungen und/oder $1,8 \%$ in Zeitarbeitsverhältnissen. ${ }^{10}$ Zudem sind hier merkliche Unterschiede zwischen Männern und Frauen festzustellen. So sind deutlich mehr Männer (73,9\%) als Frauen (56,7\%) in Normalarbeitsverhältnissen beschäftigt gewesen. Ein hoher Anteil von Frauen war in Teilzeit beschäftigt (26,8\%), während nur 3,4\% der Männer in Teilzeit arbeiteten. Und Frauen (12,6\%) waren gut

3 Die historische Bedeutung des Normalarbeitsverhältnisses hat darin bestanden, die Risiken und Gefahren der Lohnarbeiterexistenz zu minimieren und ein Mehr an sozialer Sicherheit für die (männlichen) Erwerbstätigen und an Planungssicherheit für die Unternehmen herzustellen. In der Geschlechterforschung ist verschiedentlich darauf hingewiesen worden, dass in Deutschland das Normalarbeitsverhältnis freilich nur für Männer ohne Migrationshintergrund ,normal“ gewesen ist (vgl. dazu etwa Aulenbacher 2006; Bührmann 2010).

4 Nicole Mayer-Ahuja (2011) hat zuletzt diese Chronologie hinterfragt und festgestellt, dass auch schon vor diesem Zeitpunkt eine beträchtliche Vielfalt von Erwerbskonstellationen zu beobachten gewesen sei und die wichtigste Veränderung in der Herausbildung von sich „konfligierenden Normen der Arbeitsregulierung" bestanden habe.

5 Darunter fallen z. B. Teilzeit- und Leiharbeit, geringfügige Beschäftigung, Scheinselbstständigkeit, Heim- und Telearbeit, befristete Arbeitsverträge und Niedriglohnbeschäftigung.

6 Für die folgenden Zahlen und Daten vgl. Wingerter 2009.

7 Gemeint sind damit die Erwerbstätigen im Alter von 15 bis 64 Jahren, die nicht in Bildung oder Ausbildung sind.

$811 \%$ waren selbstständig tätig. Die Gesamtsumme ist kleiner als $100 \%$, da die mithelfenden Familienangehörigen hier nicht berücksichtigt worden sind.

9 Das Statistische Bundesamt setzt hier allerdings nur eine Wochenarbeitszeit von 20 oder weniger Stunden an.

10 Hier ergibt sich eine Summe höher als 22,2\%, da Kernerwerbstätige sowohl befristet und in Teilzeit oder als Leiharbeiter bzw. Leiharbeiterin geringfügig beschäftigt sein können. 
Abb. 1: Abhängig Beschäftigte nach abhängigen Erwerbsformen in Mio., unterschieden nach Normalarbeitsverhältnissen (NAV) und atypischen Beschäftigungsverhältnissen (ATV). (Quelle: Statistisches Bundesamt; eigene Darstellung)

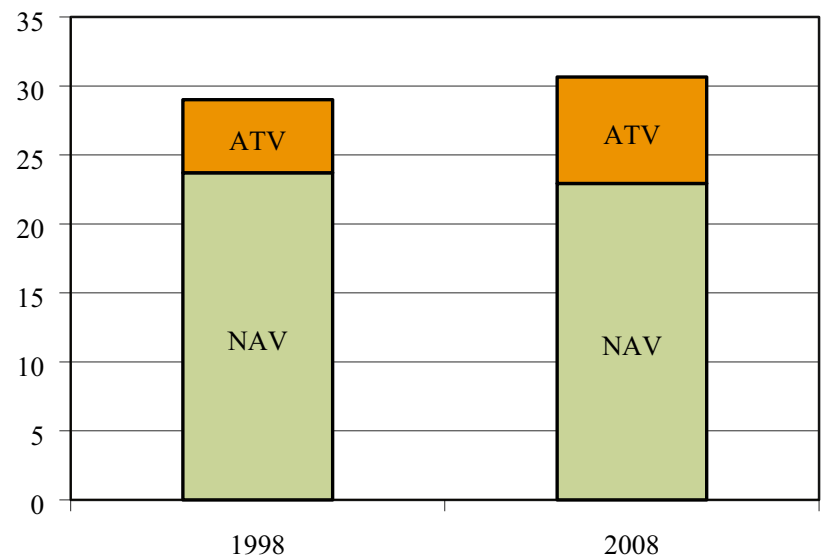

viermal häufiger als Männer $(3,0 \%)$ geringfügig beschäftigt. Markante Unterschiede lassen sich auch in Bezug auf den Bildungsabschluss feststellen: Je niedriger der Bildungsabschluss ist, desto eher sind Personen in atypischen Beschäftigungsformen tätig. 35,9\% der Kernerwerbstätigen in atypischen Beschäftigungen hatten keine anerkannte Berufsausbildung, nur 14,6\% besaßen einen Hochschulabschluss.

Die angesprochene Erosion des Normalarbeitsverhältnisses führt dazu, dass immer mehr Menschen in sogenannten prekären Verhältnissen leben und sich in einem ,heiklen Schwebezustand“ zwischen Wohlfahrt und Armut befinden. Der Begriff „Prekarität“ dient in arbeitssoziologischen Studien dazu, die Transformation der Arbeitsgesellschaft und ihre Auswirkungen auf die sozialen Arrangements von Arbeit zu erforschen. Mithilfe dieses Begriffs sollen also nicht bestimmte Arbeitsverhältnisse bewertet oder gar verurteilt werden (vgl. etwa Wingerter 2009), sondern einerseits die subjektiven Verarbeitungsformen einer Erosion des Normalarbeitsverhältnisses und andererseits auch deren gesellschaftsheoretischen Konsequenzen und praktischen Vermittlungen erforscht werden.

Pierre Bourdieu (2004) hat schon früh hervorgehoben, dass das Prekariat „überall““ ist: Es taucht sowohl im privaten, aber auch im öffentlichen Sektor weitgehend unabhängig etwa von Geschlecht, Ethnizität und Alter auf. Im Zuge dieser sozialen Entgrenzung der Prekaritätsrisiken können - so macht Mario Candeias (2008, S. 122) deutlich - vielfältige Formen der Prekarisierung beobachtet werden, die ,je nach Klassenzugehörigkeit, geschlechtlichen, ethno-nationalen oder anderen Zuschreibungen ganz unterschiedlich ausfallen und verschieden bearbeitet werden“. Diese Vielfalt an Prekarisierungsformen legt eine allgemein gehaltene Begriffsfassung nahe, wie sie z. B. Arne Kalleberg (2009, S. 2) vorschlägt: „By ,precarious work' I mean employment that is uncertain, unpredictable, and risky from the point of view of the worker".

Ähnlich wie schon beim Unternehmerbegriff findet sich nun auch in Bezug auf den Prekaritätsbegriff eine ausweitende Verkürzung. Fokussieren sich die wirtschaftssoziologischen Studien bei einer Ausweitung des Unternehmerbegriffs zunächst auf das erfolgreiche Unternehmertum, so konzentriert man sich in arbeitssoziologischen Debatten bei der Ausweitung des Prekaritätsbegriffs auf die Erforschung von Prekarisierungsprozessen bei abhängigen Beschäftigungsformen. So setzen etwa die theoretischen Überlegun- 
gen von Robert Castel (2000; siehe auch Castel und Dörre 2009) zur „Zone der Prekarität“ bei den objektiven Folgen sozialstaatlicher Deregulierung für abhängig Beschäftigte an. Die empirischen Studien von Klaus Dörre et al. (2004, 2006) richten sich auf deren subjektive Verarbeitungsformen von Prekarität. Darauf zielen z. B. auch die Forschungsbemühungen von Franz Schultheis und seinem Forschungsteam. Im Sinne einer qualitativen Gesellschaftsanalyse geht es ihnen darum, „die auf subjektiver Ebene identifizierbaren Wahrnehmungs-, Bewertungs- und Bewältigungsweisen des arbeitsweltlichen Wandels mittels verstehender Interviews zu erforschen" (Schultheis 2010, S. 203) und so die Dynamiken der Prekarisierung und deren milieuspezifischen Formen der Unsicherheitsbewältigung auszuloten (vgl. Pelizzari 2009). Beide Forschungsteams interessieren sich dafür, wie Menschen damit umgehen, dass sie nicht wissen, wo sie in Zukunft leben und als was sie arbeiten werden, sie nicht für längere Zeit planen können und sie beständig mit der Angst leben müssen, wenn nicht heute, dann vielleicht doch morgen erwerbslos $\mathrm{zu}$ werden. Mit Blick darauf werden ausgehend von der besonderen Relevanz von Erwerbsarbeit weitere Dimensionen in die Analysen mit einbezogen. Zuletzt hat etwa Klaus Kraemer (2008) zwischen den Untersuchungsdimensionen Erwerbsstelle, Erwerbsverlauf und Lebenslage unterschieden und zugleich vorgeschlagen, systematischer zwischen Prekarität im Sinne einer negativen statistischen Abweichung von den sozialen Normalstandards eines geschützten Arbeitsverhältnisses sowie einer subjektiv wahrgenommenen, „gefühlten“ Prekarität zu unterscheiden. Diese Unterscheidung biete die Möglichkeit, die Auswirkungen von Prekarisierung nicht nur an den Rändern der Arbeitsgesellschaft auszuloten, sondern auch in ihrem Kern.

Festzuhalten bleibt zunächst: Obwohl in den arbeitssoziologischen Debatten immer wieder auch für einen weiten Prekaritätsbegriff plädiert worden ist, konzentriert man sich bisher auf die Erforschung von Prekarisierungsprozessen abhängiger Erwerbsarbeit. Dabei werden mit Blick auf die Erosion des Normalarbeitsverhältnisses insbesondere drei Momente diskutiert: erstens, welche Gruppen sind von der Erosion betroffen, zweitens, inwiefern verändern sich die Formen der Beschäftigtenverhältnisse und, drittens, welche objektiven und subjektiven Folgen haben diese atypischen Beschäftigungsverhältnisse für die Lebenslage und die Lebensführung der Betroffenen.

\section{Die Erosion des Normalunternehmertums und die Prekarisierung selbstständiger Erwerbsarbeit}

Aktuelle Befunde zeigen, dass auch das einleitend schon erwähnte Normalunternehmertum spätestens seit den 1980er Jahren - also etwas später als das Normalarbeitsverhältnis - zu erodieren scheint. Dies betrifft insbesondere drei Bestimmungsmomente des Normalunternehmertums: die Person des Unternehmers bzw. der Unternehmerin, die Formen und die Intensität des unternehmerischen Arbeitens sowie die Folgen für die Lebensführung und -lage unternehmerischer Personen. Die ersten beiden Momente sind mittlerweile gut belegt und werden in der Forschung schon breit diskutiert:

Erstens zeigt sich immer deutlicher, dass nicht mehr nur vor allem berufserfahrene, erwerbstätige Männer ohne Migrationshintergrund ein Unternehmen gründen. Zwar orientiert sich in Deutschland noch immer das unternehmerische Leitbild am männlichen 
Normalunternehmertum, aber seit 1999 schließt sich der „gender gap“ in Bezug auf die Gründung, Weiterführung oder Übernahme von Unternehmen. So ist 2008 und 2009 die Differenz der TAE (,total entrepreneurial activities“) zwischen Gründerinnen und Gründern nicht mehr statistisch signifikant. Diese Entwicklung geht aber nicht auf eine Erhöhung der unternehmerischen Aktivitäten von Frauen zurück, sondern beruht darauf, dass weniger Männer ein Unternehmen gegründet haben (vgl. Sternberg et al. 2010). Dagegen gründen immer mehr Menschen mit Migrationshintergrund in Deutschland ein Unternehmen. Laut Labour Force Survey in the EU, Candidate and EFTA Countries (2008) für Deutschland hat sich von 1990 bis 2005 der Anteil der Unternehmer und Unternehmerinnen in der ausländischen Bevölkerung von sechs auf 11,8\% fast verdoppelt, d. h. von den ca. 870.000 Gründenden in Deutschland waren 2005170.000 ausländischer Herkunft. Damit hat - im Übrigen entgegen dem EU-Trend - in Deutschland der Anteil der nichtdeutschen Selbstständigen kontinuierlich zugenommen. ${ }^{11}$ Zudem ist bemerkenswert, dass sich der Anteil der Ausländerinnen, die sich selbstständig gemacht haben, seit 1985 in Deutschland mehr als verdoppelt hat. Mittlerweile gründen fast genauso viel ausländische wie deutsche Frauen in Deutschland Unternehmen. Mit Blick auf diese Zahlen kann deshalb gesagt werden: Ausländische Frauen haben nicht mehr, aber auch kaum weniger Interesse an Gründungen als deutsche Frauen. Und sie werden auch nicht mehr, aber eben auch nicht weniger als andere Frauen an einer Unternehmensgründung gehindert.

Zweitens wird immer deutlicher, dass zunehmend mehr Gründungen nicht in Vollzeit, sondern vielfach im Neben- oder Teilzeiterwerb erfolgen (vgl. Piorkowsky 2004; DangelVornbäumen 2010). Laut Angaben des Statistischen Bundesamtes (2008, S. 5) stieg die Anzahl der Unternehmer und Unternehmerinnen zwischen 1996 und 2006 in Vollzeit von 3,0 Mio. auf 3,5 Mio., im Zuerwerb von 396.000 auf 631.000, und im Nebenerwerb ist ein Anstieg von 385.000 auf $516.000 \mathrm{zu}$ verzeichnen. 2006 ist zudem erstmals ein Rückgang der Zahl der Selbstständigen im Haupterwerb zu beobachten: Ihre Zahl sank gegenüber 2005 um 2,7\% auf 3,4 Mio. Demgegenüber erhöhte sich im gleichen Zeitraum die Zahl der Selbstständigen im Zuerwerb von 631.000 auf 770.000 und im Nebenerwerb von 516.000 auf 543.000.

Aktuelle Zahlen zeigen zudem, dass immer weniger Selbstständige neben ihren eigenen weitere Arbeitsplätze schaffen, sie gleichwohl aber nicht als Scheinselbstständige zu betrachten sind: Die Zahl dieser sogenannten Solo-Unternehmer, ${ }^{12}$ bei denen die unternehmerische Arbeitgeber- und Arbeitsfunktion zusammenfallen, ist in den letzten Jahren stetig gestiegen, sodass ihre Anzahl die der Unternehmerinnen und Unternehmer mit Beschäftigten überschritten hat: Laut Statistischem Jahrbuch von 2009 (Statistisches Bundesamt 2009, S. 89) sind in Deutschland im Jahr 2007 etwa 12\% aller Erwerbstätigen selbstständig gewesen. Das entspricht 4,16 Mio. Selbstständigen. Diese Zahl hat sich

11 Da erst seit 2005 vom Statistischen Bundesamt der Migrationshintergrund ermittelt wird, werden die Zahlen in Bezug auf die Nationalität angegeben. Der hier erkennbare Trend einer kontinuierlichen Steigerung der Gründungen durch Personen mit Migrationshintergrund dürfte aber derselbe sein.

12 Sergio Bolonga (2006) spricht von abhängigen Arbeitenden der zweiten Generation, während z. B. Candeias (2008) oder auch Dieter Bögenhold und Uwe Fachinger (2010) von Solo-Selbstständigen sprechen. 
zwar gegenüber dem Jahr 2000 um 14\% erhöht, jedoch geht diese Erhöhung fast ausschließlich auf die Zunahme von Solo-Unternehmern zurück, also denjenigen, die keine Beschäftigten haben: Während nämlich die Anzahl der Unternehmerinnen und Unternehmer mit Beschäftigten um 2\% anstieg, nahm sie bei den Solo-Unternehmern um $26 \%$ zu.

Anders als die beiden ersten Bestimmungsmomente sind die Lebensführungen und -lagen der Selbstständigen bisher noch kaum erforscht, und erst langsam wird deutlich, dass nicht nur viele Unternehmer und Unternehmerinnen erfolgreich, sondern auch immer mehr prekär wirtschaften und um ihr wirtschaftliches Überleben kämpfen. Diese prekären Selbstständigen steigen gerade nicht entweder in gesicherte Verhältnisse auf oder müssen ihr Unternehmen aufgeben. Vielmehr verbleiben sie in einem „heiklen Schwebezustand“ zwischen Wohlfahrt und Armut.

\subsection{Befunde zur objektiven Prekarisierung unternehmerischen Arbeitens}

Mit Blick auf die Zunahme des Solo-Unternehmertums verwundert es zunächst nicht, dass kleine und kleinste Gründungsprojekte gegenwärtig das Gründungsgeschehen in Deutschland dominieren: Im Jahr 2010 wurde von 936.000 Personen eine selbstständige Tätigkeit begonnen (darunter 396.000 Personen Vollerwerbsgründer und damit gleich viel wie im Vorjahr), wobei 540.000 Personen dies als Nebenerwerbstätigkeit begannen, was einer Steigerung von 14\% gegenüber 2009 entspricht (KfW-Gründungsmonitor 2011, S. 1). Als Hauptstadt prekären Unternehmertums gilt Berlin. Hier waren 2007 allein 8.402 Unternehmer und Unternehmerinnen auf ergänzende Transferzahlungen aus der Grundsicherung für Arbeitslose (SGB II) angewiesen. Das entspricht mehr als $5 \%$ aller Selbstständigen in Berlin, während z. B. in Hamburg, Frankfurt a. M. und Köln der Anteil zwischen 1 und 2\% betrug (vgl. DGB Berlin/Brandenburg 2008, S. 8 f.). Im Januar 2012 ist die Zahl der Selbstständigen, die auf Transferzahlungen angewiesen sind, in Berlin auf 9.209 Personen angestiegen (vgl. Arbeitsagentur für Arbeit 2012). Viele Gründungen erweisen sich zudem als nicht nachhaltig. So sind ein Drittel der Gründenden spätestens nach drei Jahren wieder aus dem Markt ausgeschieden. Die Abbruchrate liegt bei Gründenden aus der Arbeitslosigkeit heraus mit $41 \%$ noch höher (KfW-Gründungsmonitor 2008, S. 7).

Entgegen der verbreiteten Vorstellung dominiert also gegenwärtig das Normalunternehmertum zumindest nicht das Gründungsgeschehen. Darüber hinaus zeigen einige Studien, dass unter den etablierten Selbstständigen eine starke Einkommensspreizung und damit auch ausgeprägte soziale Ungleichheitsverhältnisse zu beobachten sind (vgl. Bögenhold und Fachinger 2010): Joachim Merz (2006) hat auf der Grundlage der Einkommensstatistik $^{13}$ errechnet, dass Unternehmer bzw. Unternehmerinnen 2003 im Durchschnitt über ein jährliches Nettoeinkommen von $38.562 €$ verfügten, die abhängig Beschäftigten

13 Relativierend ist hier allerdings anzumerken, dass die Einkommensstatistiken für Selbstständige weniger zuverlässige Aussagen zulassen als für abhängig Beschäftigte, denn Selbstständige können sich Einkommen nach eigenem Ermessen auszahlen. Zudem betrachten einige ihre Einnahmen gleichzeitig als Einkommen, und andere wiederum bilden aus den Einnahmen zunächst Rücklagen und zahlen sich dann erst ein Einkommen aus. Im Großen und Ganzen dürften diese statistischen Probleme aber nichts am Trend der Befunde ändern. 
verfügten im Durchschnitt über ein Einkommen von 22.480€. Damit besaßen Selbstständige ein um ca. $40 \%$ höheres Nettoeinkommen als abhängig Beschäftigte. Betrachtet man jedoch die Zahlen genauer, dann zeigt sich eine bemerkenswerte Polarisierung der Einkommen aus unternehmerischer Arbeit, denn die Hälfte der Unternehmer und Unternehmerinnen verdiente weniger als $14.252 €$ im Jahr. Die Unterschiede verschärfen sich nochmals, wenn man die einkommensstärksten mit den einkommensschwächsten Selbstständigen vergleicht. Die einkommensstärksten $10 \%$ verdienten mehr als das 100-fache der einkommensschwächsten 10\% der Selbstständigen. Damit hat sich 2003 mehr als die Hälfte der Unternehmer und Unternehmerinnen entweder an oder schon unterhalb der Armutsgrenze befunden. Da viele der solchermaßen unternehmerisch Tätigen aber über andere Einkommensquellen verfügten, ist die statistisch ausgewiesene Armutsquote der Haushalte von Selbstständigen geringer. Laut Sozioökonomischem Panel (SOEP) lag sie in den Jahren 2001 bis 2006 bei etwa 10\% (Statistisches Bundesamt 2008, S. 168). Dieser Wert gehört freilich noch zu den höchsten unter den Haushalten mit Vollzeit-Erwerbstätigen: „Nur die Gruppe der an- und ungelernten Arbeiter/innen liegt mit 13,3\% in 2001 und 18,1\% in 2006 deutlich über diesem Wert, bei den qualifizierten Angestellten beträgt die Armutsquote nur 1,8\% in 2001 und 4,0\% in 2006" (Pongratz und Simon 2010, S. 35). ${ }^{14}$

Allerdings sind nicht alle Unternehmer und Unternehmerinnen gleichermaßen von Prekaritätsrisiken betroffen: Dieter Bögenhold und René Leicht (2000) haben schon früh darauf hingewiesen, dass offenbar klassische Professionen wie Ärzte, Rechtsanwälte, aber auch Apotheker aufgrund ihrer berufsständischen Strategien privilegierte Einkommensansprüche erhalten haben, während andere Berufsgruppen, die infolge der Ausweitung des sogenannten Dienstleistungssektors - wie z. B. Unternehmensberater oder Trainer - vermehrt auftauchen (Resch 2005), dies für sich (noch?) nicht bewerkstelligen konnten. So erfordern zwar Berufe wie Übersetzer, Journalisten und Dolmetscher eine akademische Ausbildung, aber dies hat nicht notwendigerweise zur Folge, dass sie auch regelmäßig ein Einkommen über der Armutsgrenze sichert (vgl. dazu auch Fachinger 2002). Bögenhold und Fachinger (2010) machen zudem darauf aufmerksam, dass speziell Solo-Selbstständige von Prekarität betroffen sind. Besonders hoch aber ist das Prekaritätsrisiko unter Solo-Unternehmerinnen. Das Forschungsteam um Claudia Gather zeigt auf Grundlage einer Sekundäranalyse der Berliner Einkommensstatistik von 2005, dass rund zwei Drittel aller Unternehmerinnen in Berlin ein Einkommen von monatlich unter $1.250 €$ brutto bezogen. Für ganz Deutschland ermittelten Gather et al. (2010, S. 105), dass $40 \%$ der Unternehmerinnen über ein Bruttoeinkommen von monatlich 1.278€ verfügen und so kaum von ihrem Einkommen leben, geschweige denn andere davon (mit) ernähren können.

\subsection{Befunde zur subjektiv gefühlten Prekarisierung unternehmerischen Arbeitens}

Die skizzierten Befunde verdeutlichen: Einige Selbstständige haben zwar ein sehr hohes Einkommen, jedoch leben nicht wenige an oder unterhalb der Armutsgrenze, und viele

14 Allerdings ist positiv zu vermerken, dass sich die Armutsquote bei den Selbstständigen zwischen 2001 und 2006 nicht weiter erhöht hat, sondern im Unterschied zur Gesamtentwicklung stabil geblieben ist. 
befinden sich in einer Zone der Prekarität. Dass viele Unternehmer und Unternehmerinnen aber nicht nur objektiv mit einem niedrigen Einkommen, sondern auch subjektiv mit Sinnverlusten, Anerkennungsdefiziten und Planungsunsicherheiten - mit einer subjektiv „gefühlten Prekarisierung“ - zu kämpfen haben, dies illustrieren die Ergebnisse einer ersten Pilotstudie, die an der Universität Münster durchgeführt worden ist. ${ }^{15}$ In leitfadengestützten Interviews mit Unternehmerinnen und Unternehmern konnten unterschiedliche Typen unternehmerischer Prekarität identifiziert werden. Abbildung 2 fasst diese Typologie zusammen.

Ein erster Typus, der als ,erlebte Prekarität“ bezeichnet wird, zeichnet sich dadurch aus, dass er sich sowohl in einer sozialstrukturell objektiv als heikel beobachtbaren Lage befindet und dies auch subjektiv so erlebt. Die beiden anderen Typen unterscheiden sich in jeweils einer Dimension von diesem Typus. Der Typus ,empfundene Prekarität“ empfindet subjektiv, dass er sich in einer prekären Lage befindet, aber objektiv scheint sein Einkommen auf längere Sicht nicht unter den gesellschaftlichen Standard zu sinken (Typus II). Der Typus „gelebte Prekarität“ empfindet dagegen seine Lage subjektiv nicht als prekär, sie ist aber objektiv durchaus prekär (Typus III). Zum besseren Verständnis dieser Typologie wird im Folgenden je ein Fallbeispiel für jeden Typus vorgestellt.

Dem Typus I der „erlebten Prekarität“ entspricht eine Unternehmerin, die einen Olivenölhandel betreibt. Frau T. ist Mitte 20 und hat zunächst BWL studiert, dann aber einen Studienfachwechsel zur Ethnologie vollzogen. Sie importiert jährlich etwa drei bis vier Tonnen Olivenöl, das sie von einem griechischen Bauern einkauft, in ihrem Keller in Flaschen abfüllt und an der Haustür wiederum verkauft. Ihre Kundschaft findet sie durch Mund-zu-Mund-Propaganda. Sie hat aber auch schon mit dem bekannten TV-Koch Johann Lafer im Fernsehen gekocht und ihr Olivenöl vermarktet. Ihre Kundinnen und Kunden haben sogar Verständnis für längere Wartezeiten, denn dies erhöht - so erläutert Frau T. - den authentischen Charakter des Produkts, kommt ihre Kundschaft doch zu ihr wegen der „,Geschichte hinter dem Produkt“. Frau T. erklärt: „Die Geschichte gehört dazu, dass dem Kunden das Spaß macht, das zu kaufen, also das, was du halt im Supermarkt nicht mehr hast, dass du einfach so ein bisschen quatschst.“

Das Unternehmen ist im Grunde eine Ausgründung des Unternehmens, das ihre Mutter in Rheinland-Pfalz seit Ende der 1990er Jahre betreibt. Als das Interview geführt wird, überlegt Frau T. gerade, ob sie eine wissenschaftliche Karriere anstreben oder aber sich verstärkt um ihr Unternehmen kümmern soll, denn von ihrem Verdienst aus dem Olivenhandel allein kann sie nicht leben, sondern ist auf andere Einkommensquellen angewiesen. Zugleich empfindet sie selbst ihre aktuelle Situation als prekär und sucht nach einer Lösung: „Wenn man diesen Schritt machen wollen würde, das richtig professionell zu machen, dass man auch davon lebt, dann müsste man erst mal das deutlich teurer machen oder halt andere Möglichkeiten finden, vielleicht noch mal richtige Feinschmeckerläden

15 Insgesamt sieben Interviews mit prekär unternehmerisch Tätigen sind im Wintersemester 2009/2010 und Sommersemester 2010 an der Universität Münster im Kontext des Lehrforschungsprojektes „Prekäres Unternehmertum“ durchgeführt worden. Im Anschluss wurden weitere zehn Interviews geführt. Sie wurden im Stil der Grounded Theory ausgewertet. Teile der Ergebnisse wurden im Juni 2010 gemeinsam aufgearbeitet und der Öffentlichkeit vorgestellt. 
Abb. 2: Typologie prekären Unternehmertums
Objektive Prekaritätslage

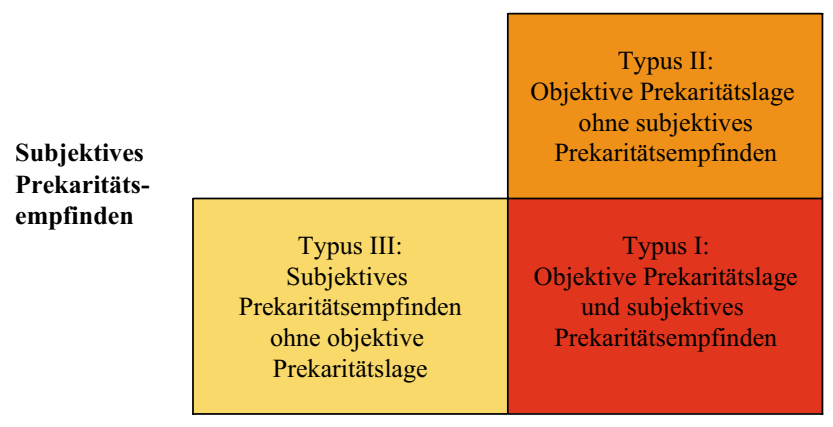

dann auftreiben, in denen man dann halt ganz kleine Mengen für ganz viel Geld verkauft, aber dann muss ich auch sagen, das ist so eine Strategie von Werbung, die ich blöd finde.“

Ihrer Meinung nach hat die unternehmerische Tätigkeit zwar Vorteile bei ihren Bewerbungen, aber der Zeitaufwand stehe in keinem adäquaten Verhältnis zum Gewinn. So sieht sie derzeit auch keine Grenzen mehr zwischen Arbeit und Freizeit. Dieses entgrenzte Arbeiten belastet Frau T. einerseits, andererseits macht ihr aber ihre Arbeit auch Spaß. Sie versteht ihre Arbeit als wichtige Unterstützung für griechische Bauern und kann sowohl ihre BWL-Kenntnisse als auch ihr Wissen aus dem Ethnologiestudium einbringen. Anders als andere prekär wirtschaftende Unternehmerinnen und Unternehmer empfindet sie ihre Lage also auch nicht als aussichtslos, sondern betrachtet ihre derzeit gelebte unternehmerische Prekarität als Sprungbrett in eine gesichertere Zukunft.

Die ledige Theaterpädagogin Frau S. dagegen erklärt: ,ich verdiene, glaube ich, ganz gut so, ich bin total zufrieden damit“. Sie empfindet also ihre Situation subjektiv nicht als prekär, vor ,allem weil sie von allen Seiten Jobs“ bekommt. So teilt sie mit: „Ich hatte Glück dadurch, dass ich auch schon früh angefangen hab mit der Arbeit und schon so lange [...] in der Theaterszene bin, dass ich sofort Kontakte hatte und ein Netzwerk und Leute auf mich zukamen, die sagten: Ah hast Du Lust [...] hier und da mitzumachen [...], dass ich nie, ähm, Eigeninitiative zeigen musste, mich irgendwo zu bewerben.“

Gleichwohl weiß Frau S. um ihre objektiv prekären Einkommensverhältnisse und sagt dann auch: „Du weißt halt nie, was am Ende des Jahres rum kommt oder was in einem halben Jahr is, und da merkt man schon, also das kriegt man schon mit, ich habe keine Sicherheit, so, ich bin immer, ich muss mich immer auf Sachen verlassen, und da kann man sich zwischendurch schon mal Sorgen machen, so einfach vom Gefühl her.“

Frau S. hat ein kulturwissenschaftliches Studium absolviert und arbeitet seit drei Jahren als freie Theaterpädagogin. Typisch scheint hier zu sein, dass sie sich - wie viele andere im Bereich der Kultur- und Kreativwirtschaft - mit relativ wenig Einkommen zufrieden geben muss, obgleich sie über einen akademischen Abschluss verfügt. Sie hat im letzten Jahr ein Bruttoeinkommen von monatlich etwa $1300 €$ erwirtschaftet. ${ }^{16}$ Zugleich ist sie im Falle von Krankheit, Alter und auch Arbeitslosigkeit kaum abgesichert. Deshalb fragt

16 Damit liegt Frau S. knapp über dem von Gather et al. (2010) ermittelten Durchschnittseinkommen Berliner Unternehmerinnen. 
sich Frau S. auch, wie sie eine Familien gründen kann, zumal sich für sie zwischen ihren einzelnen Projekten und Honorarverträgen kaum finanzielle Spielräume ergeben: „Ich wollte schon immer Kinder haben - so. Und es kann sein, dass das mit der Selbstständigkeit nicht reicht [...]. Mit dem Geld und mit der Arbeit, und dass doch regelmäßig Geld so reinkommt."

Frau S. empfindet also ihre aktuelle Situation nicht als prekär und ist somit ein Beispiel für den Typus der gelebten Prekarität. Perspektivisch sieht sie allerdings Probleme insbesondere im Hinblick auf die Umsetzung ihres Kinderwunsches.

Kann Frau S. als Beispiel für den Typus II dienen, so handelt es sich bei Herrn O. um ein charakteristisches Beispiel für den Typus III, der ,gefühlten Prekarität“. Herr O. ist zum Zeitpunkt des Interviews 55 Jahre alt und hat keine Berufsausbildung absolviert. Er besitzt seit einigen Jahren ein Taxiunternehmen, in dem etwa 20 Fahrer tätig sind. Herr O. stammt aus Asien, lebt aber seit über 20 Jahren in Deutschland und ist mit einer Deutschen ohne Migrationshintergrund verheiratet. Beide haben zwei erwachsene Söhne. Einer der Söhne führt offiziell das Unternehmen und arbeitet auch im Unternehmen mit. Herr O. gibt an, dass er und einer seiner Söhne am Tag oft mehr als 12 Stunden Taxi fahren. Er findet es gut, sein eigener Chef zu sein. Mit seinem Verdienst ist Herr O. zufrieden; obwohl er ihn nicht näher erläutern will, sagt er: „Wer sich nicht dumm anstellt und wach bleibt, verdient schon ganz gut". Ja, er verdient so gut, dass er regelmäßig Fernreisen nach Asien und Nordamerika unternehmen kann. Trotzdem empfindet Herr O. seine Lage als prekär und erklärt, dass seine Frau „zur Sicherheit“ als Sprechstundenhilfe bei einem Arzt arbeitet, „um in harten Zeiten eine zweite Stütze zu haben“, und berichtet von gesundheitlichen Problemen in seiner Familie. So scheint dann auch die gefühlte Prekarität vorherrschend. Denn Herrn O. plagen massive Zukunftssorgen:

„Aber eine Firma haben ist halt nicht so leicht, wie man sich das vorstellt. Du musst Unternehmenssteuern zahlen und für das Finanzamt und die Steuerprüfung ein durchsichtiges Rechnungswesen machen. Du bist eigentlich ständig unter Druck. Da kriegst du manchmal nicht regelmäßig Geld. Eine ständige Berg- und Talfahrt [...], wie man so sagt. Du machst dir Sorgen, eigentlich immer. Bei Tag und bei Nacht. Du hast böse Albträume und so weiter. Auch wenn's gut läuft, machst du dir Sorgen, dass es vielleicht bald nicht mehr so gut läuft. Und wenn's schlecht läuft, denkst du, wenn's bald nicht besser läuft, dann ist alles bald vorbei."

Mit Blick auf die hier diskutierten Befunde zur objektiven Prekarität und subjektiv gefühlten Prekarisierung unternehmerischen Arbeitens kann ein Unternehmertum als prekär gelten, wenn sich die unternehmerisch Tätigen objektiv an der Armutsgrenze und/ oder sich subjektiv in einer als heikel empfundenen sozialen Lebenslage befinden und ihre Lebensführung entsprechend ausrichten (müssen), d.h., wenn das Einkommens-, Schutz- und Inklusionsniveau auf längere Sicht unter den gesellschaftlichen Standard zu sinken droht bzw. sinkt und/oder die unternehmerisch Tätigen darauf hoffen müssen, unternehmerisch erfolgreich zu sein, und doch permanent befürchten, (noch) weiter sozial abzusteigen. Prekäres Unternehmertum wird also - wie auch in den arbeitssoziologischen Studien über abhängige Erwerbstätige - sowohl mit Blick auf objektive Erwerbsstrukturen wie Marktbedingungen als auch auf subjektive Orientierungen bzw. Handlungsstrategien bestimmt. 


\subsection{Ursachenkomplexe prekären Unternehmertums}

Was sind nun die Ursachen für prekäres Unternehmertum? Hans Pongratz und Stefanie Simon (2010) haben in einem Überblicksaufsatz zum bisherigen Forschungsstand u.a. drei zentrale Ursachenkomplexe identifiziert: Prekäres Unternehmertum kann demnach erstens als Folge „unzureichender Marktanpassungen“ entstehen, sich zweitens als „Konsequenz aus einem lebensphasenspezifischen Arrangement" ergeben oder aber drittens in „unternehmerischen Lebens- und Familienmodellen“ begründet sein.

Prekäres Unternehmertum entsteht für Pongratz und Simon am häufigsten als Folge unzureichender Marktanpassungen. Diese werden in der Forschung vor allem in der sogenannten Gründungs- und Nachgründungsphase von Unternehmen beobachtet. So stellen von den neu gegründeten Unternehmen in den ersten vier Jahren gut $40 \%$ den Betrieb wieder ein (Brüderl et al. 2007, S. 301 ff.). Die Gründe für mangelnden unternehmerischen Erfolg sind vielfältig: Genannt werden u. a. defizitäre Branchen- und Marktkenntnisse, mangelhafte unternehmerische Planungen, unzureichende Finanzierung, unrealistische Umsatzerwartungen, mangelnde unternehmerische Erfahrung oder auch ungeeignete fachliche Voraussetzungen.

In den letzten 20 Jahren ist mit Blick auf diese Probleme in Deutschland wie in anderen EU-Staaten eine umfangreiche Beratungs- und Förderinfrastruktur entstanden. Sie soll dazu dienen, die angesprochenen Probleme abzubauen, die Startvoraussetzungen der Gründenden zu verbessern und ihre Kompetenzen zweckrationaler unternehmerischer Entscheidungen zu stärken. Trotz derartiger Unterstützungen bleibt die Gründung, Übernahme oder Weiterführung eines Unternehmens oft ein riskantes Wagnis, das mit erheblichen Unwägbarkeiten verbunden ist. Denn für eine meist unbestimmte Zeit kann nur mit niedrigen und unsicheren Einnahmen bei erheblichen Kosten gerechnet werden. Die hier beschriebene Situation unzureichender Marktanpassung kann aber auch in späteren Unternehmensphasen insbesondere durch Veränderungen oder Krisen am Markt entstehen. Selbstständige haben vielfältige Reaktionsmöglichkeiten: So können sie neue Produkte entwickeln oder Konkurrenzprodukte imitieren, sie können über eine Optimierung ihres Vertriebs und des Marketings das Image und die Bekanntheit der eigenen Produkte steigern oder aber auch die Produktionskosten durch Rationalisierungsmaßnahmen senken. Doch all diese Reaktionen implizieren neben der Erfolgsunsicherheit zunächst immer erst neue Kosten. Pongratz und Simon (2010, S. 38 f.) sehen hier deshalb die Gefahr einer „Prekarisierungsspirale“: „Die prekäre Situation unzureichender Erträge gibt Anlass zu neuerlichen Investitionen, welche den erzielbaren Überschuss kurzfristig weiter mindern - und damit die Prekarität erhöhen (bevor auf längere Frist Erfolge erwartbar sind). Anhaltender Misserfolg am Markt zwingt zur Entscheidung, entweder den Markt zu verlassen, um weitere Verluste zu vermeiden, oder noch höhere Risiken einzugehen in der Hoffnung auf Verbesserung künftiger Marktchancen“. Mehr und mehr Unternehmerinnen und Unternehmer scheitern zwar und wenden sich dann anderen Beschäftigungsmöglichkeiten zu oder gründen ein neues Unternehmen. Sie werden zu Re-Startern (Kranzusch und Kay 2007). Jedoch gibt es zunehmend Unternehmensgründungen, denen auch nach Jahren (noch) keine wenigstens einigermaßen profitable Marktanpassung gelungen ist und die wie in den Anfangszeiten um das wirtschaftliche Überleben kämpfen. Dies scheint insbesondere Solo-Unternehmer und -Unternehmerinnen in der Kultur- und 
Kreativwirtschaft zu betreffen. Hier zeigt sich, dass neben ökonomischem Gewinnstreben oder Angst vor Arbeitslosigkeit ein wesentliches Gründungsmotiv der Wunsch nach unabhängiger Lebensgestaltung und selbstbestimmten Arbeitsbedingungen ist (siehe z. B. Müller-Jentsch 2005; KfW-Gründungsmonitor 2009; Brixy et al. 2009). Diese Motivation reklamiert ja auch die Theaterpädagogin Frau S. in dem oben geschilderten Fall. Sigrid Betzelt und Karin Gottschall (2005, S. 289) haben in ähnlichen Fällen eine „,spezifische Mischung von Privilegierung und Prekarisierung" konstatiert, und Alexandra Manske (2007) stellt eine „Prekarisierung auf hohem Niveau“ fest. Deshalb verwundert es nicht, dass Künstler und Künstlerinnen zu den ärmsten Unternehmern in Deutschland zählen und gleichzeitig als „Modernisierungsavantgarde für prekäre Unternehmensformen“" (Dangel-Vornbäumen 2010) gelten.

Prekäres Unternehmertum kann aber auch - wie etwa im Falle der erwähnten Olivenhändlerin Frau T. - als Folge eines „lebensphasenspezifischen Arrangements“ (Pongratz und Simon 2010) entstehen. Dabei werden die eigenen unternehmerischen Aktivitäten als Passage betrachtet, die für eine bestimmte Phase des Erwerbslebens als Alternative zur abhängigen Beschäftigung gewählt wird. Ein solches (auch) wertrational motiviertes Unternehmertum zeichnet sich durch einen stark explorativen Charakter aus - und birgt damit höhere Risiken in sich als ein unternehmerisches Engagement mit längerfristiger Zielsetzung. Unternehmertum als Übergang ist aber insbesondere in den Lebensphasen des Berufseinstiegs und des Wiedereinstiegs nach einer freiwilligen oder unfreiwilligen, z. B. durch Arbeitslosigkeit bedingten Erwerbspause zu beobachten. So handelt es sich etwa für Rechtsanwälte oder Journalisten, Übersetzer oder Architekten für eine wichtige Einstiegsoption in den Arbeitsmarkt nach dem Studium. Aber viele Frauen versuchen auch nach einer familiär bedingten Erwerbspause (z. B. zur Betreuung der Kinder oder zur Pflege von Angehörigen) über eine selbstständige Tätigkeit wieder Zugang zum Arbeitsmarkt zu erhalten. Immer mehr Frauen machen sich auch während einer Phase selbstständig, in der sie sich um die Betreuung von Angehörigen kümmern. Sie gründen Unternehmen, weil sie keine Chance haben, nicht unternehmerisch tätig zu sein. Denn vielfach kann selbstständiger Erwerb zeitlich und räumlich variabler gestaltet werden als abhängige Erwerbsarbeit. Zugleich besteht jedoch auch die Gefahr, dass das unternehmerische Engagement wegen anderweitiger Verpflichtungen gebremst wird (vgl. Piorkowsky 2004). Zudem gründen immer mehr Menschen Unternehmen aus der (drohenden) Arbeitslosigkeit heraus. So war laut KfW-Gründungsmonitor 2009 ein überproportionaler Anteil der Gründenden zum Zeitpunkt der Unternehmensgründung arbeitslos, nämlich $20 \%$ aller Gründenden und sogar 32\% der Vollzeit-Gründenden - bei einem Anteil von nur $7 \%$ Arbeitslosen im Untersuchungssample (KfW-Gründungsmonitor 2009, S. 43).

Schließlich verweisen Pongratz und Simon (2010) auf eine dritte Ursache prekären Unternehmertums: nämlich das Unternehmertum als Lebens- und Familienmodell. Hier stehen weniger allein ökonomische Motivationen im Vordergrund, sondern es geht z. B. um den Wunsch, einen Familienbetrieb weiterzuführen oder zu übernehmen. Aus diesem Grund dürfte auch der Sohn des Taxifahrers O. in den Betrieb seines Vaters eingestiegen sein und das Unternehmen offiziell führen. Dass sich so die Prekaritätsrisiken dauerhaft erhöhen können, zeigt zum Beispiel auch eine Studie von Barbara Lemberger (2007). Anhand der Fallstudie einer kleinen Limonadenfabrikation macht sie deutlich, dass prekäres Unternehmertum nicht nur vorübergehend aufgrund einer marktbedingten Krise oder als 
lebensphasenspezifisches Arrangement akzeptiert wird, sondern von der untersuchten Familie als Dauerzustand hingenommen wird. Diese traditionalen Motive zur Gründung von Unternehmen lassen sich besonders in den Bereichen der sogenannten alten Selbstständigen wie z. B. in Landwirtschaft und Handwerk finden. Denn nicht wenige werden aufgrund der Erbfolge zu Bauern bzw. Bäuerinnen oder aufgrund des Todes des Partners bzw. der Partnerin zu Inhaberinnen bzw. Inhabern von Handwerksbetrieben. Der Erwartungsdruck und die innere Anspruchshaltung machen es schwer, ein ererbtes Unternehmen aufzugeben, und erhöhen zugleich die Bereitschaft, prekäre Erwerbsbedingungen über lange Zeit hinweg zu akzeptieren. Dies haben Heide Inhetveen und Mathilde Schmitt (2010) eindrucksvoll in einer Longitualstudie für Bäuerinnen gezeigt.

Die bisher benannten Ursachenkomplexe konzentrieren sich auf die mehr oder minder deutlich zweckrational, traditional oder auch wertrational motivierten Entscheidungen derjenigen, die prekär unternehmerisch tätig sind. Manche Prekarisierungsprozesse gehen aber gerade nicht auf ,falsche“ oder „unangemessene“ unternehmerische Entscheidungen zurück. ${ }^{17}$ Sie sind vielmehr anderen, sozialen Konstellationen geschuldet. Darauf hat Sergio Bologna (2006) aufmerksam gemacht und rückt dabei besonders eine Gruppe von Unternehmern und Unternehmerinnen in den Forschungsfokus: nämlich die ,Selbständigen zweiter Generation“"18, die er von der geschützten freiberuflichen Tätigkeit und auch der Lohnarbeit abgrenzt. Diese Selbstständigen entwickeln laut Bologna einen Habitus, dessen zentrales Merkmal es ist, dass sie nicht auf längere Sicht sicher planen können: ${ }^{19}$ Im Gegensatz zu den Lohnabhängigen sind die selbstständig Arbeitenden nämlich permanent auf dem Markt, denn ihr Angestelltenstatus hört in dem Moment auf, in dem die jeweils letzte Dienstleistung abgewickelt bzw. das Produkt abgeliefert ist. Sie können zwar z. T. über lange Zeit ohne Einkünfte bleiben, aber leben dann ,,von ständig zu schaffenden Rücklagen, um den ,Leerzeiten“ in der Arbeit zu begegnen. Der Begriff ,Risiko“ ist der Mentalität der unabhängigen Arbeit eingeschrieben“ (ebd., S. 38). Deshalb enthält die Arbeitsleistung auch stets einen „Werbeaspekt“, über den diese Selbstständigen versuchen, entweder das bestehende geschäftliche Verhältnis fortzuschreiben oder aber neue Geschäftsbeziehungen zu erschließen. Die Angst vor zu wenigen Aufträgen führt zudem dazu, dass sie sich stets der Marktnachfrage ausgeliefert sehen. ${ }^{20}$ Bologna verweist zudem darauf, dass unternehmerische Akteure, insbesondere aber die Selbstständigen zweiter Generation, den Verlust zahlreicher sozialer Schutzrechte zu beklagen haben. Sie sind vielfach z. B. von der Arbeitslosen- und Rentenversicherung ausgeschlossen, und für ihre Kranken-, Unfall- und Pflegeversicherung müssen sie weitgehend selbst sorgen. Zudem

17 Offen ist allerdings, ob es sich hier tatsächlich immer um „Entscheidungen“ handelt und nicht auch um Stressreaktionen, ungeliebte Kompromisse oder die Effekte des ganz normalen alltäglichen unternehmerischen Chaos (vgl. auch Wienold 2010).

18 Schon 1980 hat sich Gerd Vonderach (1980) mit der Gruppe der neuen Selbstständigen auseinandergesetzt, die ein Unternehmen gründen, weniger um Profit zu erwirtschaften, als sich selbst zu verwirklichen.

19 Die Auswirkungen dieser prekären Arbeitssituation hat Richard Sennett (1998) eindringlich beschrieben.

20 Bei diesen Überlegungen orientiert sich Bologna an den Thesen Theodor Geigers (1932), der selbstständig Arbeitende als Proletaroide bezeichnet. 
haben sie auch kein Anrecht auf Mindestlöhne oder gesetzliche Arbeitszeitregelungen. So erwachsen aus ihren Vergütungen keine Ansprüche an den Staat, sondern andersherum führen diese zur Verpflichtung an den Staat, Steuern zu zahlen. Da diese Steuerzahlungen an gesetzlich festgelegte Fristen gebunden sind, zugleich aber die Auftraggeberinnen und Auftraggeber allzu oft säumig sind, führt dies häufig nicht nur zu Unternehmensinsolvenzen, sondern auch zur Prekarisierung der betroffenen Selbstständigen. So mutiert für viele Selbstständige der neuen Generation die soziale Frage zu einer Steuerfrage.

Verschärfend können dann noch zwei Dinge hinzutreten: Zum einen unterliegen diese Selbstständigen zwar formal keinerlei Kontrollen oder Anweisungen von Vorgesetzten, gleichwohl geraten sie praktisch oft in die Lage, den komplexen Zwängen von Lieferketten ausgesetzt zu sein, die sie von den unternehmerischen Entscheidungen anderer abhängig machen, egal wie gut sie sich hier den Märkten angepasst haben. Zum anderen erschweren solchermaßen individualisierte Geschäftsbeziehungen zwischen Selbstständigen und Auftraggeberinnen wie Auftraggebern nicht nur kollektive Organisationsformen, ${ }^{21}$ sondern eben auch das Aushandeln ,angemessener“, mindestens aber existenzsichernder Honorare. Diese durch mehr oder minder blockierte Märkte induzierten Prekarisierungsgründe hat Candeias (2009) exemplarisch für das Unternehmen Nike aufgeschlüsselt, in dem ca. 8.000 Beschäftigte in Management-, Forschungs-, Designund Marketingabteilungen tätig sind, die Produktion jedoch von ca. 75.000 Arbeiterinnen und Arbeitern durchgeführt wird, die von unabhängigen Subunternehmen unter Vertrag genommen worden sind..$^{22}$

\section{Theoretische Konsequenzen und methodologische sowie methodische Herausforderungen aktueller Prekarisierungsprozesse von Arbeit}

Die Rekonstruktion des aktuellen Forschungsstandes zum prekären Unternehmertum und seinen Ursachen zeigt: In Deutschland arbeiten seit Beginn der 1970er Jahre nicht nur immer mehr Menschen in prekären Beschäftigungsverhältnissen. Vielmehr sind auch seit Anfang der 1980er Jahre immer mehr Menschen unternehmerisch tätig. Im Rahmen dieser Verunternehmerung der Erwerbstätigen arbeiten zudem immer mehr Personen unter prekären Bedingungen. ${ }^{23}$ Dabei lassen sich - wie skizziert - verschiedene Typen und Ursachenkomplexe prekären Unternehmertums unterscheiden. Angesichts der konstatierten Verunternehmerungs- und Prekarisierungsprozesse des Arbeitens drängt sich die Frage auf, ob und ggf. inwiefern die skizzierte Erosion des Normalunternehmertums mit der inzwischen breit diskutierten Erosion des Normalarbeitsverhältnisses verknüpft ist. Denn der Rückgang der Normalbeschäftigung ist von einer Zunahme prekären Unternehmertums begleitet, und andersherum scheint die Abnahme des Normalunternehmertums

21 Hier stellt sich die Frage, ob Parallelen zwischen dem Auftauchen der leitenden Angestellten (vgl. Hartmann et al. 1973) und der prekären Selbstständigen bestehen.

22 Ähnliche Tendenzen zeigen sich schon seit einigen Jahren bei der Vertragsproduktion in der deutschen Automobilindustrie, aber auch schon früher in der ländlichen Kontraktproduktion des Südens (vgl. Wienold 2007, 2010).

23 Auf die Prozesse der innerbetrieblichen Verunternehmerung gehe ich an dieser Stelle nicht ein. 
mit einer Zunahme prekärer abhängiger Arbeitsverhältnisse verbunden. So werden in zahlreichen Branchen und Berufsgruppen mittlerweile viele Tätigkeiten nicht mehr von abhängig Beschäftigten oder - wie man lange in den Gewerkschaften mutmaßte - von Scheinselbstständigen, sondern immer öfter von Solo-Unternehmern wahrgenommen. Dieser Trend findet sich vor allem in der anwachsenden Kultur- und Kreativindustrie. 2009 waren hier nach Schätzungen des Bundesministeriums für Wirtschaft und Technologie (2010) etwa 236.837 Unternehmen am Markt aktiv. Damit ist die Zahl der Selbstständigen und Unternehmen in der Kultur- und Kreativwirtschaft im Vergleich zu 2008 um ca. 1,7\% angestiegen. ${ }^{24}$ Von diesen zählen etwa 225.000 zur Gruppe der Kleinstunternehmen - dies entspricht $97 \%$ der Unternehmen in der gesamten Kultur- und Kreativwirtschaft -, und etwa 5.300 Unternehmen gehören zur Gruppe der Kleinunternehmen. Nur bei etwa $1 \%$ der Unternehmen handelt es sich um mittelständische und Großunternehmen, die zusammen auf 1.375 Unternehmen kommen (ebd., S. 28). Kurz: Die Kultur- und Kreativwirtschaft ist - jedenfalls zurzeit - wirtschaftlich in weiten Teilen durch Kleinstunternehmen und Freiberufler geprägt. Und viele davon wirtschaften eben prekär. Darauf verweisen - wie schon angedeutet - seit einigen Jahren unterschiedliche arbeitsund professionssoziologische Studien: Alexandra Manske $(2007,2008)$ hat dies für die ITBranche gezeigt, während sich Cornelia Koppetsch und Günter Burkart (2002) mit der Werbebranche beschäftigt haben. Karin Gottschall und ihr Bremer Forschungsteam (vgl. etwa Gottschall und Betzelt 2007; Schnell 2007) untersuchten dies für Alleindienstleistende in Kulturberufen und Caroline Dangel-Vornbäumen (2010) für künstlerische Berufe.

Die hier exemplarisch diskutierte zunehmende Prekarisierung der unternehmerischen Erwerbstätigkeit kann auch darauf zurückgeführt werden, dass immer mehr Menschen aus der bestehenden oder drohenden Erwerbslosigkeit heraus ein Unternehmen gründen oder gegründet haben; denn mit der Umstrukturierung vom aktiven zum aktivierenden Wohlfahrtsstaat im Zuge der Hartz-IV-Reformen wurden Arbeitssuchende und Arbeitslose auch dazu aufgefordert, sich selbstständig zu machen. So ist die Förderung der unternehmerischen Selbstständigkeit in den vergangenen Jahren zu einem immer wichtigeren Handlungsfeld der Wirtschafts-, aber auch der Sozialpolitik aufgestiegen. Besonders hervorzuheben ist hier neben der Lockerung der Handwerksordnungen die Förderung von Existenzgründungen aus der Erwerbslosigkeit. ${ }^{25}$ Beide Interventionen (be-)fördern einen

24 Die Unternehmenslandschaft entwickelte sich damit etwas schwächer als in den Vorjahren. Dies machen die Veränderungsraten der Vorjahre deutlich: 2007 2,9\%, 2008 3,1\% (vgl. Bundesministerium für Wirtschaft und Technologie 2010, S. 28). Aber auch für 2010 ist von einer Steigerung der Zahlen auszugehen (vgl. Bundesministerium für Wirtschaft und Technologie 2012).

$25 \mathrm{Ab}$ dem 28.12.2011 sind von der Bundesagentur für Arbeit jedoch die Mittel zur Förderung unternehmerischer Tätigkeiten reduziert und die Kriterien verschärft worden: Ein sogenannter Gründungszuschuss kann demnach geleistet werden, wenn die Personen bis zur Aufnahme der selbstständigen Tätigkeit Anspruch auf Entgeltersatzleistungen nach SGB III haben oder in einer Arbeitsbeschaffungsmaßnahme nach dem SGB III beschäftigt gewesen sind. Beim Start der selbstständigen Tätigkeit müssen Gründende noch einen Restanspruch auf Arbeitslosengeld von mindestens 150 Tagen haben, und dessen Dauer darf nicht allein auf §127 Abs. 3 SGB III beruhen. Außerdem müssen sie die notwendigen Kenntnisse und Fähigkeiten zur Ausübung der geplanten selbstständigen Tätigkeit darlegen. Dabei muss eine fachkundige Stelle - in der Regel entweder Industrie- und Handelskammern, Handwerkskammern, berufsständische Kam- 
kontinuierlichen Anstieg der Zahl der Unternehmerinnen und Unternehmer und insbesondere der Solo-Unternehmer.

Dabei ist allerdings wohl nicht von einer linear zunehmenden Prekarisierung vom Normalunternehmertum bis hin zu prekären abhängigen Beschäftigungsverhältnissen im Sinne einer negativen Karriere - auszugehen, denn sowohl abhängig als auch selbstständig Arbeitende sind von objektiver wie subjektiver Prekarisierung betroffen. Mit Blick auf diese Prekarisierungsprozesse der abhängigen und selbstständigen Erwerbsarbeit ergibt sich ein äußerst komplexes und zum Teil widersprüchliches Bild, dessen Vermittlungsverhältnisse wie Bestimmungsmomente dringend zu erforschen sind. Dabei ist es wichtig, nicht nur die abhängigen Formen von Arbeit, sondern auch die selbstständigen systematisch zu untersuchen. Es gilt zu erforschen, inwiefern etwa die Prekarisierung abhängiger Arbeit eine Prekarisierung selbstständiger Arbeit und umgekehrt nach sich zieht. Zudem ist zu untersuchen, ob möglicherweise Prekarisierungsprozesse Prozesse der Normalisierung überlagern könnten. Schließlich sollten die Übergänge vom Normalarbeitsverhältnis zum Normalunternehmertum und solche von prekären Arbeitsverhältnissen zum prekären Unternehmertum analysiert werden.

Bei der Auseinandersetzung mit diesen Forschungsfragen geht es freilich nicht nur um eine Historisierung, sondern auch um eine gesellschaftstheoretisch angeleitete Kontextualisierung der Verunternehmerungs- wie Prekarisierungsprozesse des Arbeitens. So gilt es zu fragen, ob und ggf. inwiefern mit Blick auf diese hier diskutierten Verunternehmerungstendenzen unter den Beschäftigten und den damit verknüpften Prekarisierungsprozessen der für kapitalistische Gesellschaften grundlegende Widerspruch zwischen Kapital und Arbeit implodiert und welche Konsequenzen daraus erwachsen könnten. Erwartet uns z. B. ein Gesellschaftsmodell, in dem nicht mehr wie noch im Fordismus primär große und mittlere Unternehmen, sondern kleine und kleinste Unternehmen das Akkumulationsregime dominieren, sodass die unternehmerische Flexibilität immer weiter vorangetrieben und die Arbeit immer stärkeren Deregulierungs- wie Fragmentierungsprozessen unterworfen werden? Mit dieser Frage hat sich auch Sergio Bologna intensiv am Beispiel Italiens auseinandergesetzt. Er stellt fest, dass viele der prekären Selbstständigen - Bologna spricht wie gesagt von den „Selbständigen der zweiten Generation“ - ehemals in einem Normalarbeitsverhältnis tätig gewesen sind und staatlicherseits gefördert bzw. gefordert nun prekär unternehmerisch arbeiten. Das könnte bedeuten, dass der Gegensatz zwischen Kapital und Arbeit nicht implodiert, sondern lediglich verschoben wird. Zugleich ist aber auch zu fragen, ob die Ausweitung des Unternehmerbegriffs nicht nur statistischen Erfordernissen, sondern auch diskurspolitischen Überlegungen gehorcht. Wenn die Betroffenen der Prekarisierungsprozesse nicht mehr als Arbeiter und Arbeiterinnen, sondern als Unternehmer und Unternehmerinnen angerufen werden, dann fällt ein organisierter

mern, Fachverbände oder auch Kreditinstitute - das Existenzgründungsvorhaben begutachten und die Tragfähigkeit der Existenzgründung bestätigen. Der Gründungszuschuss wird bei positiver Begutachtung in zwei Phasen geleistet. Für die erste Phase wird der Zuschuss in Höhe des zuletzt bezogenen Arbeitslosengeldes und $300 €$ zur sozialen Absicherung für sechs Monate gewährt. Für weitere neun Monate können $300 €$ pro Monat zur sozialen Absicherung gezahlt werden, wenn eine intensive Geschäftstätigkeit und hauptberufliche unternehmerische Aktivitäten dargelegt werden können (vgl. dazu ausführlich Bundesagentur für Arbeit 2012). 
Kampf gegen diese Prekarisierungsprozesse und ihre handfesten Folgen umso schwerer: Macht es doch einen Unterschied, ob Prekarisierte selbst für ihre Lage verantwortlich gemacht werden (können), weil sie zum Beispiel die „falschen“ Entscheidungen getroffen haben, sich nicht ,genügend“ als unternehmerische Persönlichkeit entwickelt haben oder aber angeblich nicht ,ausreichend“ unternehmerische Initiative zeigen, oder ob sie sich selbst als Arbeiterinnen bzw. Arbeiter betrachten, die die gesetzlichen Schutzrechte für abhängig Beschäftigte verloren haben.

Schon jetzt ist jedoch klar, dass offensichtlich nicht mehr nur Arbeitnehmer und Arbeitnehmerinnen, sondern auch eine zunehmend größer werdende Zahl von Unternehmern und Unternehmerinnen der Solidarität aller bedürfen; denn immer mehr unternehmerisch Tätige sind auf staatliche Transferzahlungen angewiesen, indes können sie bisher kaum am Wohlfahrtsstaat partizipieren - dies zeigten ja z. B. die Daten für Berlin. Sozialpolitische Studien verweisen an dieser Stelle auf die mangelnde Einbindung der Selbstständigen insbesondere in die gesetzliche Renten-, Kranken- sowie Arbeitslosenversicherung (vgl. etwa Schulze-Buschoff 2005). So sind z.B. laut aktueller amtlicher Rentenstatistik zum 31.12.2005 nur 188.131 Personen, die selbstständig im Handwerk, im Bereich der Kunst oder der Publizistik arbeiteten, gesetzlich rentenversichert gewesen (vgl. Forschungsportal der Deutschen Rentenversicherung 2012). Und zum 31.12.2010 waren lediglich 169.626 Personen in der Künstlersozialkasse versichert (Künstlersozialkasse 2012). Schließlich waren nach Angaben der Bundesregierung 2009 nur 88.816 Selbstständige freiwillig arbeitslosenversichert (vgl. Presseerklärung des Bundestages 2010). ${ }^{26}$ Dass also z. B. Frau S. nicht wirksam gegen die Risiken von Alter, Krankheit und Arbeitslosigkeit versichert ist, erscheint weniger als Sonder- denn als Regelfall.

Statt weitere Sonderregelungen - wie etwa die Künstlersozialkasse oder auch die derzeit oft diskutierte Reform des verkürzten ALG-I-Bezuges - zu implementieren, wird in der wissenschaftlichen Debatte schon länger eine universelle Einbeziehung aller in die Sozialversicherungssysteme angemahnt. Diese Forderung unterstützen inzwischen auch Teile der SPD und Bündnis 90/Die Grünen. Andere fordern neben einer Optimierung der Gründungsberatung, wenigstens die Risiken des unternehmerischen Scheiterns zu begrenzen und das Insolvenzrecht zu reformieren (vgl. Kranzusch und Kay 2007). Es gilt aber auch, die Vermittlungen zwischen sozialpolitischen Interventionen und den individuellen Umgangspraxen in den Fokus der Forschung zu rücken, ist doch davon auszugehen, dass Menschen gerade in prekären Arbeitsverhältnissen nicht mehr in Routinen handeln, sondern von Fall zu Fall entscheiden müssen und hier das Außeralltägliche zum Alltäglichen wird. Diese unternehmerischen Akteure sind gezwungen, stets innovativ zu sein. Interessant ist dann aber die Frage, wie sie es schaffen, aus ihren alltäglichen Inventionen Innovationen zu machen. Dies nachzuvollziehen ist eine wichtige Aufgabe der Sozialwissenschaften.

Aus den hier skizzierten möglichen theoretischen Konsequenzen ergeben sich einige methodologische wie methodische Herausforderungen. Zum einen macht es wenig Sinn, die Forschung auf die Beschreibung unternehmerischer Strukturen zu beschränken, ohne

26 Nach der Reform der freiwilligen Arbeitslosenversicherung für Selbstständige liegen allerdings noch keine belastbaren Zahlen vor, wie überhaupt die Datenlage hier insgesamt recht unterkomplex und kaum systematisch erforscht ist. 
angeben zu können, wie und wodurch diese Strukturen hervorgebracht und reproduziert werden. Zum anderen geht es darum, nicht nur nach den rationalen Entscheidungen der unternehmerisch Handelnden zu fragen. Vielmehr - und dies zeigen die hier vorgestellten Typen unternehmerischen Handelns und die anhand des bisherigen Forschungstandes rekonstruierten zentralen Ursachenkomplexe prekären Unternehmertums - sind Selbstständige eben nicht nur von ökonomischen Motiven getrieben, entscheiden sie nicht immer kühl kalkulierend. Wichtige weitere explizite wie implizite Motivationen können z. B. habituell verankerte traditionale Werthaltungen wie etwa familiäre (Ver-)Bindungen, aber auch wertrationale Haltungen sein, die sich z.B. in Selbstverwirklichungsansprüchen manifestieren. Insofern ist ökonomisches Handeln nicht nur immer auch soziales Handeln (Beckert und Zafirovsky 2006; Maurer 2008), sondern auch als Resultat sozial strukturierender und strukturierter Praxen zu betrachten. Deshalb ist es wenig sinnvoll, unhinterfragt von einem unvermittelten Zusammenhang zwischen Mikro- und Makroebene auszugehen und so von der Eigensinnigkeit der Individuen und ihren Identitätsausbildungsprozessen wie -praxen zu abstrahieren.

Zur Erforschung dieser Fragen erscheinen mir soziologische Praxistheorien besonders anschlussfähig, denn sie interessieren sich nicht nur dafür, dass Akteure unternehmerisch handeln, sondern auch dafür, wie sie unter welchen Bedingungen und ausgehend von welchen Konventionen ihre Aktivitäten am Markt praktisch koordinieren. Damit stehen - jenseits eines objektivierenden Strukturalismus einerseits und eines subjektivierenden Individualismus andererseits - die empirisch-konkreten Praxen ${ }^{27}$ der unternehmerischen Akteure und die damit verbundenen Wahrnehmungs-, Erlebens- und Deutungsmuster im Forschungsfokus. Die Relevanz dieser doppelten, d.h. subjektiven und objektiven Perspektive ist hier am Beispiel der unterschiedlichen Typen prekären Unternehmertums illustriert worden. Die soziologische Unternehmerforschung darf sich gerade nicht darin erschöpfen, ökonomische Gesetzmäßigkeiten oder Erklärungen für Marktmechanismen theoretisch zu konstruieren. Vielmehr hat sie als Erfahrungswissenschaft die Kontingenz des Praktischen und damit auch den subjektiv gemeinten Sinn der sozialen Akteure in den Blick zu nehmen. Es geht darum, gerade nicht von der grundlegend gegebenen Pluralität der das unternehmerische Handeln koordinierenden pragmatischen Handlungslogiken zu abstrahieren, sondern empirisch-praktisch die konventionsbasierten Koordinationsweisen und damit verknüpften institutionalisierten Organisationsformen auszuleuchten. ${ }^{28}$ Wie unternehmerische Akteure Märkte wahrnehmen, ihr Handeln angesichts dessen mit dem erwarteten Handeln anderer koordinieren, Wertigkeiten prüfen, auf Machtkonstellationen reagieren sowie Informationen nutzen, um für ihre unternehmerische Zukunft zu planen, und welche Konventionen sie dabei anleiten - all das sind pragmatische Problemstellungen in der Konkurrenz um knappe Güter und Ressourcen, denen sich die unternehmerischen Akteure stellen müssen und die sie - so oder so - bewältigen müssen.

27 Praxen werden dabei als Effekte rekursiv aufeinander bezogener diskursiver und nichtdiskursiver Praktiken verstanden.

28 Ausgehend von solchen Überlegungen hat sich - anknüpfend an Bourdieus Feld- und Habituskonzeption - mittlerweile eine Ökonomie der Konventionen formiert; vgl. dazu Thévenot et al. 2005. 
Zudem implizieren Praxistheorien eine rekonstruktive Forschungshaltung. Dies bedeutet: Ökonomische Phänomene werden als sozial konstruiert und damit im Grunde kontingent begriffen. In diesem Sinne führt etwa Bourdieu (1987, S. 97) aus: „Die Theorie der Praxis als Praxis erinnert gegen den positivistischen Materialismus daran, daß Objekte der Erkenntnis konstruiert und nicht passiv registriert werden, und gegen den intellektualistischen Idealismus, daß diese Konstruktion auf dem System von strukturierten und strukturierenden Dispositionen beruht, das in der Praxis gebildet wird und stets auf praktische Funktionen ausgerichtet ist.“ Es gibt also nicht den ,normalen Unternehmer“, der im Laufe der Zeit mit immer besseren Methoden und Kategorien immer genauer erfasst werden kann. Vielmehr ist mit Michel Foucault (1973) davon auszugehen, dass in wissenschaftlichen Diskursen selbst die Gegenstände - also hier z. B. das Normalunternehmertum konstruiert werden, von denen in ihnen die Rede ist. Diese Überlegungen implizieren eine Abkehr von der Annahme, Analysen unternehmerischer Praxen seien ,nur“ als Beschreibungen von Wirklichkeit zu verstehen. Deshalb werden Forschungsbefunde systematisch auf ihre möglichen Theorieeffekte befragt. Die Rede vom Normalunternehmertum - wie im Übrigen auch vom Normalarbeitsverhältnis - und die damit eng verknüpfte Fokussierung auf die Formen der Normalbeschäftigung sind in dieser Perspektive als Theorieeffekte zu betrachten, die dazu führen, dass andere Formen unternehmerischer Praxen ausgeblendet bleiben. Es bedarf jedoch nicht nur einer Diversifizierung des unternehmerischen Leitbildes, sondern auch einer Differenzierung des Unternehmerbegriffs selbst, denn unter diesen Begriff werden ganz unterschiedliche unternehmerische Aktivitäten subsumiert. Sie unterscheiden sich nicht nur darin, ob sie erfolgreich sind oder nicht, sondern auch in Bezug auf das Ausmaß des Erfolges, die rechtliche Form des Unternehmens, die Anzahl der Beschäftigten, den Inhalt, die Ziele und den zeitlichen Umfang der unternehmerischen Aktivitäten sowie die Gründe der Unternehmensgründung und ihrer Begründungsformen. Auf diese Weise könnte auch die eingangs rekonstruierte ausweitende Verkürzung der Begriffe des Unternehmerischen wie des Prekären produktiv überwunden werden, sodass die heterogenen Formen unternehmerischer Aktivitäten wie die vielfältigen Formen der Prekarisierung in den Blick genommen, theoretisch begriffen und deren Vermittlungen analysiert werden können. Hier reicht es jedoch nicht, wie dies Candeias (2008, S. 74) fordert, den Begriff des „Solo-Selbständigen aus dem Diskurs über eine Gesellschaft von Unternehmern heraus zu nehmen [sic!]“. Vielmehr geht es darum zu fragen, weshalb dieser Begriff an welchen Stellen in diesem Diskurs auftaucht.

\section{Fazit und Perspektiven}

Worin besteht also der einleitend postulierte Mehrwert eines Dialoges zwischen Wirtschafts- und Arbeitssoziologie? Zum einen legen die Befunde der Arbeitssoziologie eine Ausweitung der wirtschaftssoziologischen Unternehmensforschung vom Normalunternehmertum auf das nichtnormale Unternehmertum nahe, denn seit den 1980er Jahren kann nicht nur eine zunehmende Verunternehmerung des Arbeitens konstatiert werden, sondern auch der Trend, dass immer weniger Unternehmer und Unternehmerinnen dem Leitbild des Normalunternehmertums entsprechen. Zum anderen aber wird deutlich, dass die Arbeitssoziologie von den bereits vorliegenden Befunden aus der Wirtschaftssozio- 
logie profitieren kann. Hier zeigt sich, dass Selbstständige eben nicht nur als „Personifizierung des Kapitals“ oder auch als bloße „Agenten“ kapitalistischer Bewegungsgesetze kühl kalkulierend primär ökonomisch motivierte Entscheidungen treffen. Vielmehr werden viele gerade nicht erfolgreiche, prekär wirtschaftende Selbstständige nicht nur von zweckrationalen, sondern eben auch wertrationalen und traditionalen Beweggründen motiviert. Dies manifestiert sich in den unterschiedlichen Typen prekären Unternehmertums. Sie werden in ihrer unternehmerischen Praxis von den kapitalistischen Bewegungsgesetzen eher getrieben, als dass sie sie antreiben. Und sie generieren mit ihren unternehmerischen Aktivitäten weniger (gesellschaftlichen) Reichtum, sondern eher ihre eigene Armut.

Durch eine solche Verknüpfung arbeits- und wirtschaftssoziologischer Debatten könnte die Unternehmensforschung insgesamt mehr über ihren Gegenstand erfahren und das heterogene Feld unternehmerischer Aktivitäten und der daran Beteiligten erforschen. Dieses Mehr an Wissen über die Praxis prekären Unternehmertums ermöglichte, erstens, ganz praktisch, dass Selbstständige, die prekär wirtschaften, besser, weil passgerechter beraten, unterstützt und gefördert werden könnten. Zweitens könnte klarer werden, weshalb Menschen sich überhaupt darauf einlassen, über einen längeren Zeitraum prekär unternehmerisch aktiv zu sein - mit Bourdieu (2005, S.7) könnte man vielleicht vom „Paradox der doxa“ sprechen. Drittens böte ein Mehr an Wissen über prekäre unternehmerische Praxen weitere Aufklärung darüber, wie die hier diskutierten Verunternehmerungs- wie Prekarsierungsprozesse gesellschaftstheoretisch einzuordnen sind: Handelt es sich wie schon in den 1920er Jahren um die Ausweitung einer Klassenfraktion, nämlich der „proletaroiden Selbständigkeit“ (Geiger 1932), formiert sich eine neue soziale Gruppe, wie z.B. analog zu den leitenden Angestellten in den 1970er Jahren, die aber politisch kaum Bedeutung erlangten, oder ist die Zunahme prekarisierten Unternehmertums als bloßes Übergangsphänomen potenziell erfolgreicher Gründungen zu betrachten? Perspektivisch gilt es allerdings, nicht nur nach möglichen Transformierungsprozessen der Produktionsverhältnisse zu fragen, sondern auch danach, ob und ggf. inwiefern sich etwa auch andere zentrale Ungleichheitsverhältnisse wie z. B. das Geschlechterverhältnis oder die Verhältnisse zwischen unterschiedlichen Ethnien wandeln und wie diese unterschiedlichen Transformationsprozesse mit Blick auf sich möglicherweise wandelnde Regulationsweisen miteinander vermittelt sein könnten.

Die Befunde einer solchen Erforschung unternehmerischer Aktivitäten lassen also auch einen wichtigen Beitrag zur Beantwortung der aktuell kontrovers diskutierten Frage erwarten, wohin die sozialen Fragmentierungen und die damit verknüpften gesellschaftlichen Dynamiken postfordistischer Gesellschaften treiben.

Danksagung: Für klärende Nachfragen und inspirierende Impulse danke ich neben den Gutachtenden dieser Zeitschrift vor allen Dingen Heinz Hartmann, Hans Pongratz und Hanns Wienold sowie den Mitgliedern des Göttinger SOFI-Kolloquiums. Die Idee zum Aufsatz entstand in dem Lehrforschungsprojekt „Prekäres Unternehmertum“ an der Universität Münster.

Open Access: Dieser Artikel unterliegt den Bedingungen der Creative Commons Attribution License. Dadurch sind die Nutzung, Verteilung und Reproduktion erlaubt, sofern der/die Originalautor/en und die Quelle angegeben sind. 


\section{Literatur}

Aldrich, H. E. (2005). Entrepreneurship. In N. J. Smelser \& R. Swedberg (Hrsg.), The handbook of economic sociology (S. 451-477). Princeton: Princeton University Press.

Arbeitsagentur für Arbeit. (2012). Statistik: aktuelle Daten für Arbeitssuchende nach Ländern - Berlin. http://statistik.arbeitsagentur.de/nn_10256/SiteGlobals/Forms/Direktsuche/ direktsuche_Form_Region.html?view=processForm\&resourceId=7658\&input_=\&pageLocale $=$ de $\&$ ste $=4 \&$ year $=2012 \&$ month $=01 \&$ category $=$ arbeitslose $\&$ topic $=$ grund lb\&region=berlin\&search=Suchen. Zugegriffen: Febr. 2012.

Aulenbacher, B. (2006). Gender meets funktionale Differenzierung. Zur mangelnden analytischen Radikalität der systemtheoretischen Verortung von Geschlecht. In B. Aulenbacher, M. Bereswill, M. Löw, M. Meuser, G. Mordt, R. Schäfer, \& S. Scholz (Hrsg.), FrauenMännerGeschlechterforschung. State of the Art (S.95-99). Münster: Westfälisches Dampfboot.

Becker, M., \& Knudsen, T. (2003). The entrepreneur as a crucial juncture in Schumpeter's work: Schumpeter's 1928 handbook entry „entrepreneur“. In R. G. Koppl, J. Birner, \& P. KurrildKlitgaard (Hrsg.), Advances in Austrian economics (Bd.6, S. 199-234). Amsterdam: Elsevier.

Beckert, J., \& Zafirovski, M. (2006). International encyclopedia of economic sociology. London: Routledge.

Betzelt, S., \& Gottschall, K. (2005). Flexible Bindungen - prekäre Balancen. Ein neues Erwerbsmuster bei hochqualifizierten Alleindienstleistern. In M. Kronauer \& G. Linne (Hrsg.), Flexicurity. Die Suche nach der Sicherheit in der Flexibilität (S. 275-294). Berlin: edition sigma.

Bögenhold, D. (1987). Der Gründerboom. Realität und Mythos der neuen Selbständigkeit. Frankfurt a. M.: Campus.

Bögenhold, D., \& Fachinger, U. (2010). Mikro-Selbstständigkeit und Restrukturierung des Arbeitsmarktes - Theoretische und empirische Aspekte zur Entwicklung des Unternehmertums. In A. D. Bührmann \& H. J. Pongratz (Hrsg.), Prekäres Unternehmertum. Unsicherheiten von selbstständiger Erwerbstätigkeit und Unternehmensgründung (S. 63-84). Wiesbaden: VS Verlag für Sozialwissenschaften.

Bögenhold, D., \& Leicht, R. (2000). „Neue Selbständigkeit“ und Entrepreneurship: moderne Vokabeln und damit verbundene Hoffnungen und Irrtümer. WSI-Mitteilungen, 53(12), 779-787.

Bologna, S. (2006). Die Zerstörung der Mittelschichten. Thesen zur neuen Selbständigkeit. Wien: Nausner \& Nausner.

Bourdieu, P. (1987). Sozialer Sinn. Kritik der theoretischen Vernunft. Frankfurt a. M.: Suhrkamp.

Bourdieu, P. (1990). Sprache und symbolische Macht. Wien: Turia + Kant.

Bourdieu, P. (2004). Prekarität ist überall. In P. Bourdieu, Gegenfeuer, Wortmeldungen im Dienste des Widerstands gegen die neoliberale Invasion (S. 107-113). Konstanz: UVK.

Bourdieu, P. (2005). Die männliche Herrschaft. Frankfurt a. M.: Suhrkamp.

Brixy, U., Hundt, C., Sternberg, R., \& Stüber, H. (2009). Unternehmensgründungen im internationalen Vergleich: Deutschland - eine Gründerwüste? IAB-Kurzbericht, 15, 1-8.

Brüderl, J., Preisendörfer, P., \& Ziegler, R. (2007). Der Erfolg neugegründeter Betriebe: eine empirische Studie zu den Chancen und Risiken von Unternehmensgründungen. Berlin: Duncker \& Humblot.

Bührmann, A. D. (2007). Das Bild vom Normalunternehmer. Deutungsmuster in der Existenzgründungsberatung. In L. W. Mayerhofer, C. Berendt, \& A. Sondermann (Hrsg.), Fallverstehen und Deutungsmacht. Akteure in der Sozialverwaltung und ihre Klienten (S. 119-142). Opladen: Barbara Budrich Verlag.

Bührmann, A. D. (2010). Gender matter, class matters, and what else? Problematisierungsweisen sozialer Ungleichheiten im erodierenden Institutionengefüge von (Sozial-)Staat, (Lohn-) Arbeit und Familie. In H.-G. Thien (Hrsg.), Klassen im Postfordismus (S. 152-173). Münster: Westfälisches Dampfboot. 
Bührmann, A. D., \& Pongatz, H. J. (2010). Prekäres Unternehmertum. Einführung in ein vernachlässigtes Forschungsfeld. In A. D. Bührmann \& H. J. Pongratz (Hrsg.), Prekäres Unternehmertum. Unsicherheiten von selbstständiger Erwerbstätigkeit und Unternehmensgründung (S. 7-24). Wiesbaden: VS Verlag für Sozialwissenschaften.

Bundesagentur für Arbeit. (2012). Finanzielle Hilfen für Existenzgründerinnen und Existenzgründer. http://www.arbeitsagentur.de/nn_26400/Navigation/zentral/Buerger/Hilfen/Existenzgruendung/Existenzgruendung-Nav.html. Zugegriffen: Feb. 2012.

Bundesministerium für Wirtschaft und Technologie. (2010). Monitoring zu wirtschaftlichen Eckdaten der Kultur und Kreativwirtschaft 2009. Berlin.

Bundesministerium für Wirtschaft und Technologie. (2012). Monitoring zu ausgewählten wirtschaftichen Eckdaten der Kultur- und Kreativwirtschaft in Deutschland 2010. Berlin.

Candeias, M. (2008). Die neuen Solo-Selbständigen zwischen Unternehmergeist und Prekarität. Prokla 150. Zeitschrift für kritische Sozialwissenschaft, 38, 65-82.

Candeias, M. (2009). Neoliberalismus, Hochtechnologie, Hegemonie. Grundrisse einer transnationalen kapitalistischen Produktions- und Lebensweise. Eine Kritik. Hamburg: Argument.

Castel, R. (2000). Die Metamorphosen der sozialen Frage. Eine Chronik der Lohnarbeit. Konstanz: UVK.

Castel, R., \& Dörre, K. (Hrsg.). (2009). Prekarität, Abstieg, Ausgrenzung. Die soziale Frage am Beginn des 21. Jahrhunderts. Frankfurt a. M.: Campus.

Dangel-Vornbäumen, C. (2010). Freischaffende Künstler und Künstlerinnen - Modernsierungsavantgarde für prekäres Unternehmertum? In A. D. Bührmann \& H. J. Pongratz (Hrsg.), Prekäres Unternehmertum. Unsicherheiten von selbstständiger Erwerbstätigkeit und Unternehmensgründung (S. 137-166). Wiesbaden: VS Verlag für Sozialwissenschaften.

DGB Berlin/Brandenburg. (2008). Berlin - Hauptstadt der prekären Beschäftigung. http://berlinbrandenburg.dgb.de/themen/++co++248b0bd6-c58d-11e0-775b-00188b4dc422. Zugegriffen: Febr. 2012.

Deutschmann, C. (2008). Kapitalistische Dynamik. Eine gesellschaftstheoretische Perspektive. Wiesbaden: VS Verlag für Sozialwissenschaften.

Dörre, K., Kraemer, K., \& Speiderl, F. (2004). Prekäre Arbeit. Ursachen, soziale Auswirkungen und subjektive Verarbeitungsformen unsicherer Beschäftigung. Das Argument, 46, 378-397.

Dörre, K., Kraemer, K., \& Speiderl, F. (2006). Prekäre Beschäftigung und soziale (Des-)Integration. Ursprünge, Konsequenzen und politische Verarbeitungsformen unsicherer Beschäftigungsverhältnisse. Jahrbuch Arbeit, Bildung, Kultur, 23/24, 9-40.

Fachinger, U. (2002). Die Selbständigen: Armutspotential der Zukunft? In S. Sell (Hrsg.), Armut als Herausforderung. Bestandsaufnahme und Perspektiven der Armutsforschung und Armutsberichterstattung (S. 87-130). Berlin: Duncker \& Humblot.

Forschungsportal der deutschen Rentenversicherung. (2012). http://forschung.deutsche-rentenversicherung.de/ForschPortalWeb/view3sp.jsp?chstatakt_VersicherteVersicherte $\% 20$ insgesamt $=5$ eb65eb6\&open \&viewName=statakt_VersicherteVersicherte $\% 20$ insgesamt\&viewCaption=Statistiken $\% 20-\% 20$ Versicherte $\% 20-\% 20$ Versicherte $\% 20$ insgesamt\#5eb65eb6. Zugegriffen: Febr. 2012.

Foucault, M. (1973). Die Archäologie des Wissens. Frankfurt a. M.: Suhrkamp.

Gather, C., Schmidt, T., \& Ulbricht, S. (2010). Der Gender Income Gap bei den Selbstständigen Empirische Befunde. In A. D. Bührmann \& H. J. Pongratz (Hrsg.), Prekäres Unternehmertum. Unsicherheiten von selbstständiger Erwerbstätigkeit und Unternehmensgründung (S. 85-110). Wiesbaden: VS Verlag für Sozialwissenschaften.

Geiger, T. (1932). Die soziale Schichtung des deutschen Volkes. Soziographischer Versuch auf statistischer Grundlage. Stuttgart: Ferdinand Enke.

Gottschall, K., \& Betzelt, S. (2007). Jenseits von Profession und Geschlecht? Erwerbsmuster in Kulturberufen. In R. Gildemeister \& A. Wetterer (Hrsg.), Erosion oder Reproduktion geschlechtlicher Differenzierungen? (S. 122-144). Münster: Westfälisches Dampfboot. 
Hartmann, H., Bock-Rosenthal, E., \& Helmer, E. (1973). Leitende Angestellte: Selbstverständnis und kollektive Forderungen. Ergebnisse einer empirischen Untersuchung. Neuwied: Luchterhand.

Inhetveen, H., \& Schmitt, M. (2010). Prekarisierung auf Dauer? Die Überlebenskultur bäuerlicher Familienbetriebe. In A. D. Bührmann \& H. J Pongratz (Hrsg.), Prekäres Unternehmertum. Unsicherheiten von selbstständiger Erwerbstätigkeit und Unternehmensgründung (S.111136). Wiesbaden: VS Verlag für Sozialwissenschaften.

Kalleberg, A. L. (2009). Precarious work, insecure workers: Employment relation in transition. American Sociological Review, 74, 1-22.

KfW-Gründungsmonitor. (2008). Gründungen in Deutschland: weniger aber besser. Frankfurt a. M.: KfW-Bankengruppe.

KfW-Gründungsmonitor. (2009). Untersuchung zur Entwicklung von Gründungen im Voll- und Nebenerwerb. Frankfurt a. M.: KfW-Bankengruppe.

KfW-Gründungsmonitor. (2011). Dynamisches Gründungsgeschehen im Konjunkturaufschwung. Frankfurt a. M.: KfW-Bankengruppe.

Koppetsch, C., \& Burkart, G. (2002). Werbung und Unternehmensberater als „Treuhänder“ expressiver Werte? Talcott Parsons' Professionssoziologie und die neuen ökonomischen Kulturvermittler. Berliner Journal für Soziologie, 12, 531-550.

Kraemer, K. (2008). Prekarität - was ist das? Arbeit. Zeitschrift für Arbeitsforschung, Arbeitsgestaltung und Arbeitspolitik, 17(2), 77-90.

Kranzusch, P., \& Kay, R. (2007). 2. Chance? Hürden und Hemmnisse bei der Umsetzung von Restarts. In Institut für Mittelstandsforschung Bonn (Hrsg.), Jahrbuch zur Mittelstandsforschung 1/2007. Schriften zur Mittelstandsforschung, (Nr. 115, S. 85-129). Wiesbaden: Deutscher Universitätsverlag.

Künstlersozialkasse. (2012). Entwicklung der Versichertenzahlen - unterteilt nach den Kunstbereichen - ab dem Jahr 1991. http://www.kuenstlersozialkasse.de/wDeutsch/ksk_in_zahlen/statistik/versichertenbestandsentwicklung.php. Zugegriffen: Feb. 2012.

Labour Force Survey in the EU, Candidate and EFTA Countries. (2008). Main characteristics of national surveys, 2008. Brüssel: Dictus Publishing.

Lemberger, B. (2007). Alles für's Geschäft. Ethnologische Einblicke in die Unternehmenskultur eines kleinen Familienunternehmens. Berlin: Lit.

Manske, A. (2007). Prekarisierung auf hohem Niveau. Eine Feldstudie über Alleinunternehmer in der IT-Branche. München: Hampp.

Manske, A. (2008). Prekarisierung auf hohem Niveau oder: Risikolage Kreativarbeit. In HeinrichBöll-Stiftung (Hrsg.), Kreativen:Wirkung. Urbane Kultur, Wissensökonomie und Stadtpolitik (S. 48-52). Berlin: Heinrich-Böll-Stiftung.

Maurer, A. (2008). Institutionalismus in der Wirtschaftssoziologie. In A. Maurer (Hrsg.), Handbuch der Wirtschaftssoziologie (S. 62-86). Wiesbaden: VS Verlag für Sozialwissenschaften.

Maurer, A., \& Schimank, U. (2008). Die Gesellschaft der Unternehmen - die Unternehmen der Gesellschaft. Gesellschaftstheoretische Perspektiven der Wirtschaft. Wiesbaden: VS Verlag für Sozialwissenschaften.

Mayer-Ahuja, N. (2011). Jenseits der ,neuen Unübersichtlichkeit“. Annäherungen an Konturen der gegenwärtigen Arbeiswelt. SOFI Arbeitspapier 2011-6. Göttingen.

Merz, J. (2006). Polarisierung der Einkommen von Selbständigen? Zur Dynamik der Einkommensverteilung und der hohen Einkommen von Selbständigen und abhängig Beschäftigten. München: Munich Personal RePEc Archive (MPRA) Paper.

Mückenberger, U. (1985). Die Krise des Normalarbeitsverhältnisses. Mitteilungsblatt der zentralen wissenschaftlichen Einrichtung ,Arbeit und Betrieb“, 11/12, 3-36.

Müller-Jentsch, W. (2005). Künstler und Künstlergruppen. Soziologische Ansichten einer prekären Profession. Berliner Journal für Soziologie, 15, 159-177. 
Pelizzari, A. (2009). Dynamiken der Prekarisierung. Atypische Erwerbsverhältnisse und milieuspezifische Unsicherheitsbewältigung. Konstanz: UVK Verlagsgesellschaft.

Piorkowsky, M.-B. (2004). Unternehmensgründungen im Zu- und Nebenerwerb: Motive, Wachstumsziele und gefühlte Restriktionen. In A.-K. Achleitner, H. Klandt, L. Koch, \& K.-I. Voigt (Hrsg.), Jahrbuch Entrepreneurship 2003/04. Gründungsforschung und Gründungsmanagement (S. 207-225). Berlin: Springer.

Pongratz, H. J., \& Simon, S. (2010). Prekarisierungsrisiken unternehmerischen Handelns. In A. D. Bührmann \& H. J. Pongratz (Hrsg.), Prekäres Unternehmertum. Unsicherheiten von selbstständiger Erwerbstätigkeit und Unternehmensgründung (S.25-61). Wiesbaden: VS Verlag für Sozialwissenschaften.

Presseerklärung des Bundestages. (2010). Bundesregierung prüft freiwillige Weiterversicherung Selbständiger in der Arbeitslosenversicherung. http://www.bundestag.de/presse/ hib/2010_03/2010_058/04.html. Zugegriffen: Feb. 2012.

Rammert, W. (2008). Technik und Innovation. In A. Maurer (Hrsg.), Handbuch der Wirtschaftssoziologie (S. 291-319). Wiesbaden: VS Verlag für Sozialwissenschaften.

Resch, C. (2005). Berater-Kapitalismus oder Wissensgesellschaft? Münster: Westfälisches Dampfboot.

Schnell, Ch. (2007). Regulierung der Kulturberufe in Deutschland. Strukturen, Akteure, Strategien. Wiesbaden: Deutscher Universitäts-Verlag.

Schultheis, F. (2010). Transformationen der Arbeitswelt: Qualitative Forschung in der Tradition der kritischen Gesellschaftsanalyse Pierre Bourdieus. In I. Götz, K. Lehnert, B. Lemberger, \& S. Schondelmayer (Hrsg.), Mobilität und Mobilisierung: Arbeit im sozioökonomischen, politischen und kulturellen Wandel (S.203-223). Frankfurt a. M.: Campus.

Schulze Buschoff, K. (2005). Von der Scheinselbstständigkeit zur Ich-AG - neue sozialpolitische Weichenstellungen? Zeitschrift für Sozialreform, 51, 64-93.

Schumpeter, J. A. (1928). Unternehmer. In L. Elster, A. Weber, \& F. Wieser (Hrsg.), Handwörterbuch der Staatswissenschaften (4.Aufl., Bd. 8, S. 467-487). Jena: Gustav Fischer.

Schumpeter, J. A. (1993). Theorie der wirtschaftlichen Entwicklung. Eine Untersuchung über Unternehmensgewinn, Kapital, Kredit, Zins und den Konjunkturzyklus. Berlin: Duncker \& Humblot.

Sennett, R. (1998). Der flexible Mensch. Die Kultur des neuen Kapitalismus. Berlin: Berlin Verlag.

Smelser, N. J., \& Swedberg, R. (2005). The handbook of economic society. Princeton: Russel Sage Foundation.

Statistisches Bundesamt. (2008). Datenreport 2008. Ein Sozialbericht für die Bundesrepublik Deutschland. Wiesbaden: Statistisches Bundesamt.

Statistisches Bundesamt. (2009). Datenreport 2009. Ein Sozialbericht für die Bundesrepublik Deutschland. Wiesbaden: Statistisches Bundesamt.

Statistisches Bundesamt. (2011). Erwerbstätige in den Volkswirtschaftlichen Gesamtrechnungen. http://www.destatis.de/jetspeed/portal/cms/Sites/destatis/Internet/DE/Presse/abisz/Erwerbstaetige, templateId=renderPrint.psml. Zugegriffen: Juli 2011.

Sternberg, R., Brixy, U., \& Hundt, C. (2010). GEM. Länderbericht Deutschland. Hannover/Nürnberg: IAB. http://doku.iab.de/presse/gem07-download.pdf. Zugegriffen: Feb. 2010.

Swedberg, R. (2009). Unternehmen. In R. Swedberg, Grundlagen der Wirtschaftssoziologie (S. 105-132). Wiesbaden: VS Verlag für Sozialwissenschaften.

Swedberg, R., \& Granovetter, M. (2001). Introduction to the second edition. In R. Swedberg \& M. Granovetter (Hrsg.), The sociology of economic life (S. 1-28). Boulder: Westview Press.

Thévenaut, L., Eynard-Duvernay, F., Faverau, O., \& Salais, R. (2005). Values, coordination, and rationality: The economy of conventions, transition and development. In A. N. Oleinik (Hrsg.), The institutional economics of Russia's transformation (S. 21-43). Aldershot: Ashgate. 
Vonderach, G. (1980). Die neuen Selbstständigen: 10 Thesen zur Soziologie eines unvermuteten Phänomens. Mitteilungen aus der Arbeitsmarkt- und Berufsforschung, 13, H. 2, 153-169.

Wienold, H. (2007). Leben und Sterben auf dem Lande. Kleinbauern in Indien und Brasilien. Münster: Westfälisches Dampfboot.

Wienold, H. (2010). Die Gegenwart der Bourgeoisie. Umrisse einer Klasse. In H.-G. Thien (Hrsg.), Klassen im Postfordismus (S. 235-286). Münster: Westfälisches Dampfboot.

Wingerter, C. (2009). Der Wandel der Erwerbsformen und seine Bedeutung für die Einkommenssituation Erwerbstätiger. In Statistisches Bundesamt (Hrsg.), Wirtschaft und Statistik (Nr. 11, S. 1080-1098). Wiesbaden: Statistisches Bundesamt.

Andrea D. Bührmann, geb. 1961. Prof. Dr., Universität Göttingen, Institut für Soziologie. Aktuelle Lehr- und Arbeitsschwerpunkte: Soziologie der Geschlechterverhältnisse, insbesondere (Trans-) Formierungsprozesse der Geschlechterverhältnisse und ihrer Differenzierungsprozesse (Diversity Studies) sowie die Erforschung des Wandels der Arbeit und ihrer Organisationsstrukturen sowie Unternehmens- und Unternehmer/innenforschung. Ausgewählte Veröffentlichungen: (mit K. Hansen, M. Schmeink und A. Schöttelndreier) Entrepreneurial Diversity. UnternehmerInnen zwischen Businessplan und Bricolage, 2007; (mit H. Pongratz, Hrsg.) Prekäres Unternehmertum: Unsicherheiten einer sich ausbreitenden Erwerbsform, 2010; Intersectionality - ein Forschungsfeld auf dem Weg zum Paradigma? Tendenzen, Herausforderungen und Perspektiven der Forschung über Intersektionalität, in: GENDER. Zeitschrift für Geschlecht, Kultur und Gesellschaft, 2009. 